\title{
Performance analysis of symmetrical and bidirectional 40 Gbps TWDM-PON employing m- QAM-OFDM modulation with multi-color LDs based VLC system
}

\author{
Meet Kumari ( $\nabla$ meetkumari08@yahoo.in ) \\ Punjabi University https://orcid.org/0000-0001-9975-1557 \\ Anu Sheetal \\ Guru Nanak Dev University \\ Reecha Sharma \\ Punjabi University
}

\section{Research Article}

Keywords: next-generation passive optical network stage 2 (NG-PON2), time and wavelength division multiplexing passive optical network (TWDM-PON), orthogonal frequency division multiplexing (OFDM), quadrature amplitude modulation (QAM), visible light communication (VLC)

Posted Date: June 23rd, 2021

DOl: https://doi.org/10.21203/rs.3.rs-372983/v1

License: (c) (1) This work is licensed under a Creative Commons Attribution 4.0 International License. Read Full License

Version of Record: A version of this preprint was published at Optical and Quantum Electronics on August 6th, 2021. See the published version at https://doi.org/10.1007/s11082-021-03108-2. 


\title{
Performance analysis of symmetrical and bidirectional 40 Gbps TWDM-PON employing m-QAM-OFDM modulation with multi-color LDs based VLC system
}

\author{
Meet Kumari ${ }^{\mathrm{a}, \mathrm{c}}$, Anu Sheetal ${ }^{\mathrm{b}}$, Reecha Sharma ${ }^{\mathrm{a}}$ \\ ${ }^{a}$ Department of Electronics and Communication Engineering, Punjabi University, Patiala, India \\ bepartment of Electronics and Communication Engineering, Guru Nanak Dev University Regional Campus, \\ Gurdaspur, India \\ 'Department of Electronics and Communication Engineering, Chandigarh University, Punjab, India
}

\section{Correspondence}

Meet Kumari, Email: meetkumari08@yahoo.in, ORCID ID: 0000-0001-9975-1557

\begin{abstract}
In this work, a full-duplex time and wavelength division multiplexing-passive optical network (TWDM-PON) system is analysed. Orthogonal frequency division multiplexing (OFDM) with m-quadrature amplitude modulation (m-QAM) is employed to improve the performance of TWDM-PON for downstream and upstream transmission. Simultaneously, multi-color (390-750 nm) laser diodes (LDs) are employed for visible light communication (VLC) using various VLC links to encourage the information rate of fiber/VLC optical network. A TWDM-PON utilizing 16-, 32- and 64-QAM OFDM with ten LDs based VLC system has been analysed for full-duplex multi-color VLC signals of the system. The impact of the LDs input current and high transmission rate in the proposed PON/VLC link has been investigated for m-QAM OFDM modulation. The results show that the 40/40 Gbps 16-, 32- and 64-QAM signals over ten $8000 \mathrm{~m} \mathrm{VLC} \mathrm{links} \mathrm{and} \mathrm{a} 50 \mathrm{~km}$ fiber link are successfully transmitted at the modulation input current of $9 \mathrm{~mA}$ under bit error rate (BER) of $3.8 \times 10^{-3}$. Also, the proposed system employing 16-, 32- and 64-QAM signals over a $10 \mathrm{~km}$ fiber and ten $10 \mathrm{~m}$ VLC links provide the maximum transmission rate of 120, 100 and $80 \mathrm{Gbps}$ respectively. Moreover, the measured error vector magnitudes (EVMs) and calculated BER values for 16-QAM downstream and upstream signals, are well below the required FEC limit than high-order modulation formats. Further, the numerical analysis of the proposed system reveals the superiority of the proposed fiber/VLC links.
\end{abstract}

Key Terms: next-generation passive optical network stage 2 (NG-PON2), time and wavelength division multiplexing passive optical network (TWDM-PON), orthogonal frequency division multiplexing (OFDM), quadrature amplitude modulation (QAM), visible light communication (VLC)

\section{Declarations:}

Funding: Not applicable

Conflicts of interest: The authors declare that they have no conflict of interest.

Availability of data and material: Not applicable

Code availability: Not applicable

Author contributions: All authors contributed to the study conception and design.

Acknowledgments: The authors are thankful to Punjabi University, Patiala, India and Guru Nanak Dev University, Amritsar, India for providing opportunity and technical support to complete the work as effective. 


\section{Introduction}

In this $21^{\text {st }}$ century, the high-capacity requirements for optical networks are rising exponentially due to the rise in recent technology such as big-data analysis, cloud computing and 4k/8k video broadcast. Therefore, the fifthgeneration $(5 \mathrm{G})$ mobile wireless communication systems are currently analysed and discussed. 5G wireless networks aim to achieve higher network capacity, seamless coverage, energy, spectrum regulation and costeffectively (Bi et al. 2019; Grover et al. 2020). To address the growing demand for high-capacity and economical transmission, optical access networks based on passive optical networks (PONs) are considered as a potential candidate for 5G-transport (Hu et al. 2020). Furthermore, the traditional Gigabit-class PON networks, such as Ethernet PON (EPON) and Gigabit PON (GPON) as standardized by IEEE and ITU-T respectively include the use of time-division-multiplexing (TDM) or wavelength-division-multiplexing (WDM) techniques. However, it is found that traditional networks do not meet the foremost carrier requirements for future worldwide fiber access. This includes management, flexibility and upgradability of bandwidth, higher capacity, maximum reuse of existing optical distribution network (ODN), higher split ratio and capacity than existing deployed standards (Lee et al. 2010). Hence, in 2012, the next-generation PON (NG-PON) based 40 Gbps time and wavelength division multiplexed PON (TWDM-PON) network has been proposed and demonstrated (Kaur et al. 2017b).

To acquire 40Gbps TWDM-PON architecture, distinct solutions for modulation formats in downlink/uplink direction for example 4-level pulse amplitude modulation (4-PAM) and duobinary as well as on-off keying (OOK) have been investigated (Yeh et al. 2014). Omella, M. et al. (Omella et al. 2008) reported 10 Gbps PON using duobinary modulation over the $11 \mathrm{~km}$ fiber link. Zhang, W. F in (Zhang et al. 2010) demonstrate 10/10 Gbps WDM-PON using differential quadrature phase-shift keying (DQPSK) modulation and upstream OOK format at $20 \mathrm{~km}$ distance. Shao, Y. et al. in (Shao et al. 2017) simulated 10/10 Gbps PON using 4-PAM modulation over fiver reach of $20 \mathrm{~km}$. In (Mandal and Patra 2017) a $10 \mathrm{Gbps}$ hybrid WDM/TDM PON utilizing differential phase shift keying with 128 ONUs over $50 \mathrm{~km}$ fiber is demonstrated. However, in NG-PON access networks, the traffic rate of beyond $100 \mathrm{Gbps}$ is required due to the requirement of various broadband multiservices (Kaur et al. 2014). Thus, for 40 Gbps NG-PON access using OOK, 4-PAM and duobinary on a single frequency in fiber reach of $20 \mathrm{~km}$ are not generally implemented owing to the fiber constraints e.g. polarization mode dispersion, complex receiver structures, chromatic dispersion (CD) as well as costlier $40 \mathrm{GHz}$ transceivers (Gill et al. 2019; Yeh et al. 2014). To achieve a higher information rate (more than 100 Gbps) cost-effectively, a quadrature amplitude modulation orthogonal frequency division multiplexing (QAM-OFDM) modulation could be effectively utilized (Selvendran et al. 2019). QAM-OFDM, a spectrally efficient modulation encodes the data on various orthogonal subcarriers. On the other hand for the different channels, TDM and WDM offer time and wavelength multiplexing techniques respectively; thus restricting several consumers to access as well as share a conventional fiber link. Aside from these multiplexing techniques, OFDM can provide features such as bandwidth allocation dynamically, a high degree of freedom and ability of software reconfigurable (Grover and Sheetal 2020; Lyu et al. 2018).

Many researchers investigated the PON systems with m-QAM-OFDM (for $\mathrm{m}=4,8,16$ and 32) modulation for long-range transmission and high data rates (Bai et al. 2020; Choudhury 2018; Ju et al. 2016; Patel and Dalal 2017; Xiao et al. 2019). Hu, Z. et al. (Hu et al. 2020) demonstrate the 10/10 Gbps OFDM-WDM-PON uisng 4QAM modulation over the transmission length of $20 \mathrm{~km}$. Bi, M. et al. (Bi et al. 2019), Zhuo, X. et al. (Zhuo et al. 2020) and Chen, Q. et al. (Chen et al. 2018) demonstrated a 10 Gbps OFDM-PON system using 16-QAM modulation over $20 \mathrm{~km}$ and to increase the transmission rate, Huang, Y. et al. (Huang et al. 2020) realized the 20 Gbps OFDM-PON system using 16-QAM modulation over the $20 \mathrm{~km}$ fiber link. Xiao, Y., and Yu, J. in (Xiao and Yu 2013) demonstrated 10 Gbps WDM radio over fiber (RoF) PON utilizing 16-QAM OFDM modulation over the $40 \mathrm{~km}$ fiber link. Yeh, C.H., and Chow, C.W. in (Yeh and Chow 2009) investigated an asymmetric 16/10 Gbps (downstream/upstream) TDM-PON system using 16-QAM OFDM modulation over the $100 \mathrm{~km}$ fiber link. Kartiwa, I. et al. (Kartiwa et al. 2013) experimentally demonstrated 20 Gbps WDM-OFDM-PON using 4/8/16/32-QAM modulation schemes over the fiber link range of $20 \mathrm{~km}$. Further, one of the challenges in the NG-PON utilizing m-QAM OFDM modulation is the expensive fiber installation in geographical constraints areas. For this, radio frequency-based wireless access technologies such as RoF, wireless fidelity (WiFi) as well as worldwide interoperability for microwave access (WiMax) are widely used. However, due to the limited capacity, restricted spectrum range and the presence of severe electromagnetic interference (EMI) radiations are prohibited in lots of areas such as aircrafts cabins and hospitals (Chen et al. 2016). Here, the most favourable, visible light communication (VLC) technology significantly utilized and integratd with the PON to resolve the issue (Anis et al. 2017; Savojbolaghchi et al. 2019; Yeh et al. 2018).

For future $5 \mathrm{G}$ wireless access networks, VLC is regarded as a prominent technology because of its protocol transparency, high reliability, high security, eco-friendly, license-free spectrum (375 nm-780 nm), infrastructure flexibility, energy efficiency and less cost. Also, as compared with traditional radio frequency (RF) wireless access, VLC has EMI-free wireless access the indoor as well as outdoor environments. Besides, VLC can be 
installed and utilized for the backup optical fiber, point-to-point (P2P) last-mile access and organization connectivity to offer the system security in the presence of geographical constraints (Mallick et al. 2020; Mohd Nor et al. 2019; Prabu et al. 2017). Since wireless communication and illumination can be obtained simultaneously, light-emitting diode (LED)-based VLC has been frequently studied (Jani et al. 2019; Ma et al. 2018; Wu et al. 2017). Chen, M. et al. (Chen et al. 2020) reported the $157.5 \mathrm{Mbps}$ data rate transmission in $2 \times 2$ multiple inputs-multiple-output (MIMO)-OFDM VLC system over $0.5 \mathrm{~m} \mathrm{VLC} \mathrm{link.} \mathrm{Li,} \mathrm{H.} \mathrm{et} \mathrm{al.} \mathrm{(Li} \mathrm{et} \mathrm{al.} \mathrm{2015)}$ reported the $682 \mathrm{Mbps}$ data rate transmission over 1m VLC link in VLC system employing OFDM modulation. Also, Lu, M. et al. (Lu et al. 2019) and Deng, R. et al. (Deng et al. 2018), reported the faithful transmission at 30 Mbps and 3.63 Gbps information rate over $3.5 \mathrm{~m}$ VLC link respectively OFDM-VLC system. Chow, C.W. et al. (Chow et al. 2013) reported the successful 6.14Mbps data rate transmission over the $2 \mathrm{~m}$ VLC link in OFDMVLC system. Zhang, J. et al. (Zhang et al. 2018), experimentally revealed the reliable 1.87 Gbps data rate transmission over a $0.8 \mathrm{~m}$ VLC link in VLC-WDM system employing OFDM. Chi, N., and Shi, J. in (Chi and Shi 2015) experimentally demonstrated a 1.6 Gbps VLC system utilizing OFDM modulation over 1.1 m VLC link. Moreover, in (Mukherjee et al. 2020) a full-duplex 12/10 Gbps hybrid OFDM based free-space and wireless-over-fiber transport system over $50 \mathrm{~km}$ bidirectional fiber as well as $12 \mathrm{~m}$ downstream free-space link and $8 \mathrm{~m}$ upstream wireless link has been demonstrated. In (Mallick et al. 2018), a bidirectional wireless-overfiber based on OFDM employing polarization multiplexing technique at 10 Gbps along with 6.25 Gbps for OFDM downlink transmission and 5 Gbps along with 2.5 Gbps OFDM uplink transmission over $50 \mathrm{~km}$ fiber along with $10 \mathrm{~m}$ downstream and $5 \mathrm{~m}$ upstream wireless links is successfully demonstrated. In (Mandal et al. 2018) a bidirectional WDM hybrid fiber-wireless/fiber-wired/fiber-VLC/fiber-invisible VLC (IVLC) at 10 Gbps/50GHz milimeter wave (MMW), $10 \mathrm{Gbps} / 100 \mathrm{GHz}$ MMW over $50 \mathrm{~km}$ fiber along with $10 \mathrm{~m}$ RF and 40 $\mathrm{m}$ optical wireless link in downstream transmission is demonstrated. Also, the upstream data at $2.5 \mathrm{Gbps}$ is transmitted over $50 \mathrm{~km}$ fiber and $10 \mathrm{~m}$ free-space transmission link successfully. However, as the increasing of information rate and transmission range, an LED-based VLC system encounters heavy traffic because of the extremely finite modulation bandwidth of LEDs and the divergence of light (He et al. 2016b). However, multiinput multi-output (MIMO) technology has been generally studied in (Alqahtani et al. 2019; Deng et al. 2012; Guerreiro et al. 2020; Saraereh et al. 2019)during previous few years and applied to various wireless OFDM systems as it can improve the reliability and capacity of the systems. But there are still major issues like channel correlation, hardware impairments and implementations, modulation and interference management which need to be address for future based wireless systems (Lu et al. 2014). Besides this the integrated power line communication (PLC)-VLC system also act as an economical and efficient backbone network but it has some issues like low transmission speed, disturbance sensitivity, channels' cross-modulation, nonlinear distortions, huge size and high cost of inductors as well as capacitors utilized in PLC system (Fusheng et al. 2016). On the other hand, the laser diode (LD) based VLC systems show high pumping efficiency, modulation bandwidth, coherency and high power for point-to-point communication. Hence, the positive features of PON utilizing OFDM modulation with LDs based VLC system have attracted numerous researchers' attention towards hybrid wired/wireless links. Recently, various works on OFDM using LD based VLC system as visible laser light communication (VLLC) have been investigated and analyzed. Wei, Y. et al. (Wei et al. 2017), presented the transmission of $2.5 \mathrm{Gbps}-10 \mathrm{GHz}$ data in fiber-VLLC system utilizing OFDM over $20 \mathrm{~km}$ wired and $8 \mathrm{~m}$ VLLC link. Shi, J. et al. (Shi et al. 2018), demonstrated the successful transmission at 3.2 Gbps information rate in OFDM/offset QAM (OFDM/OQAM) on the basics of VLLC-WDM system over $50 \mathrm{~km}$ wired and $4.5 \mathrm{~m}$ VLLC links. However, these fiber-VLLC systems using a single LD are applicable for short range access networks at low transmission rate. Therefore, LDs of different wavelengths with a high output power and modulation bandwidth are regarded one of the preferred solution for constructing a long-range and high-speed VLC system. It could be of interest to study an energy-efficient NG-PON based TWDM-PON using m-QAM-OFDM modulation using multi-color LDs based VLC system. Although numerous previous work have been demonstrated on the VLLC-OFDM system, but no one work studied the TWDM-OFDM PON performance with the multi-color LDs based VLC system.

Thus, in this work, a symmetrical as well as full-duplex four wavelengths TWDM-PON using m-QAM OFDM modulations with multi-color LDs based VLC system is proposed as well as analysed. Here, TWDMPON system employing downstream and upstream wavelengths (four pairs) are engaged within $15 \mathrm{GHz}$ bandwidth where each OFDM band is modulated at 16-, 32-, and 64-QAM formats. Also, in the system coherent detection is utilized to offer supreme frequency selectivity at receiver. For the moment, the multi-color LDs based VLC system is utilized as an optimal solution for constructing a next-generation fiber-VLC system. Here, in Section 2, the proposed system architecture is presented. In Section 3, the numerical analysis of the proposed PON/VLC link under fiber impairments is illustrated. Section 4 illustrates results and discussion followed by the conclusion in Section 5.

\section{System architecture}


Fig. 1 presents the architecture of TWDM-OFDM PON with the multi-color LDs based VLC system. The incoming signal from an optical line terminal (OLT) passed through a fiber cable followed by a remote node (RN) is employed to allot the incoming signals to the four no. of optical network units (ONUs). The wired (fiber) and wireless (VLC) applications are obtained by linking optical fiber and through the wireless VLC channels by utilizing multi-color LDs (390-750 nm) in the ceiling (Chen et al. 2016).

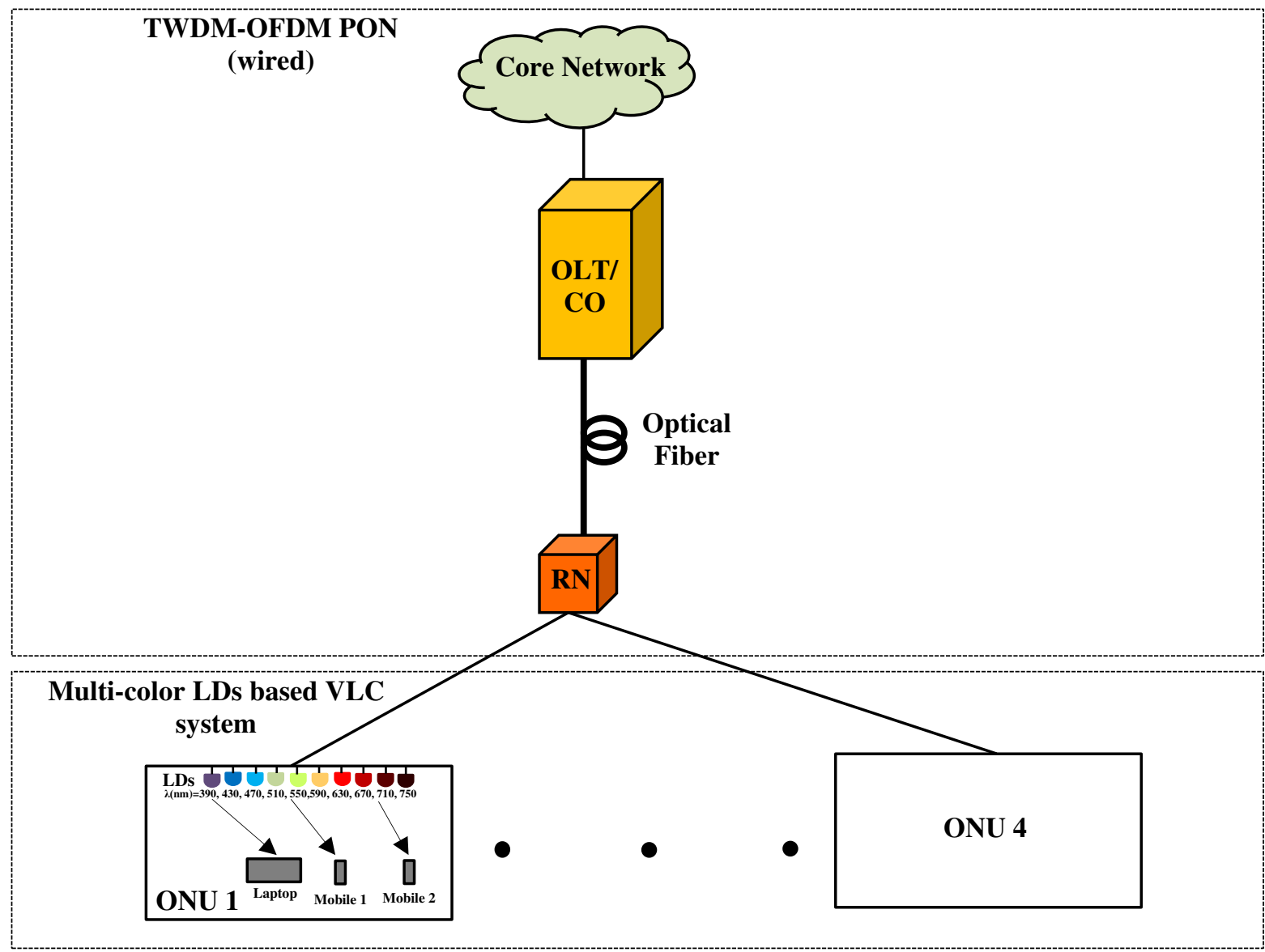

Fig. 1 Semantic diagram of the proposed TWDM-OFDM PON system employing multi-color LDs VLC system

The proposed architecture of the NG-PON2 based bidirectional 40Gbps (4×10Gbps) TWDM-PON (1:8 split ratio) using m-QAM OFDM modulation using the LD based VLC system is illustrated in Fig. 2. At OLT, four

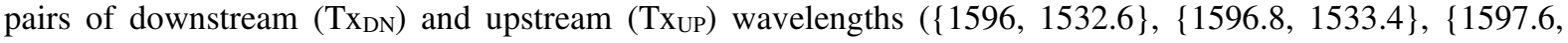
$1534.2\}$ and $\{1598.4,1535\})$ in $\mathrm{nm}$ (channel spacing=0.8 $\mathrm{nm}$ ) used for bidirectional transmission in the system.

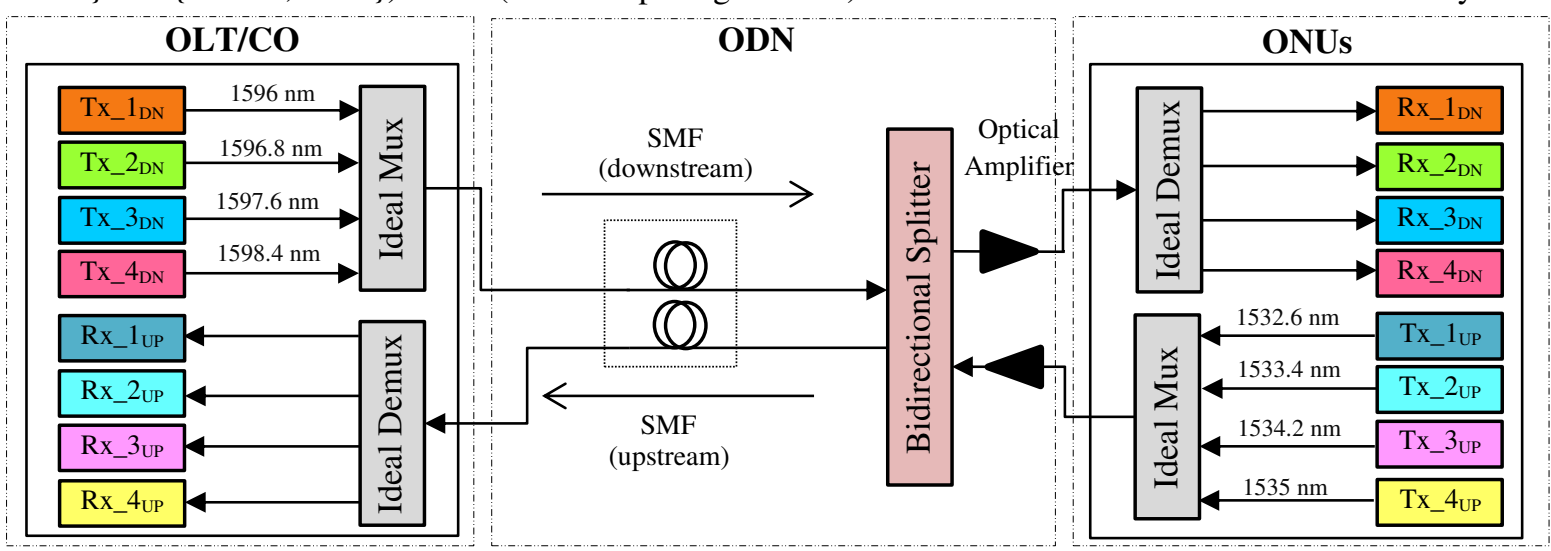

Fig. 2 Schematic diagram of full-duplex 4×10 Gbps TWDM-OFDM PON with LDs based VLC system 
In Fig. 2, for downlink, four downlink wavelengths with OFDM signals are multiplexed through ideal multiplexer, directed through a single-mode fiber (SMF) and passed to a passive splitter for downstream transmission. After amplification by using an optical amplifier (OA), the downlink signals are distributed to ONUs using an ideal de-multiplexer (Lin et al. 2015). At the users' side, the receiver chooses its definite wavelength to decode the OFDM signal. Then VLC signals are generated by multi-LDs based VLC system per OFDM wavelength. Ten colors set of LDs are utilized per VLC system to construct a wireless system. In the uplink direction, each ONU passed boosted OFDM signals are firstly de-multiplexed as well as decoded and then passed through multi-color LDs based VLC links per OFDM signal. The m-QAM modulation formats tend to transmit $100 \mathrm{Gbps}$ and more data over a channel but also suffers from fiber impairments which hinders the faithful wired/wireless distance (Yeh et al. 2014). 


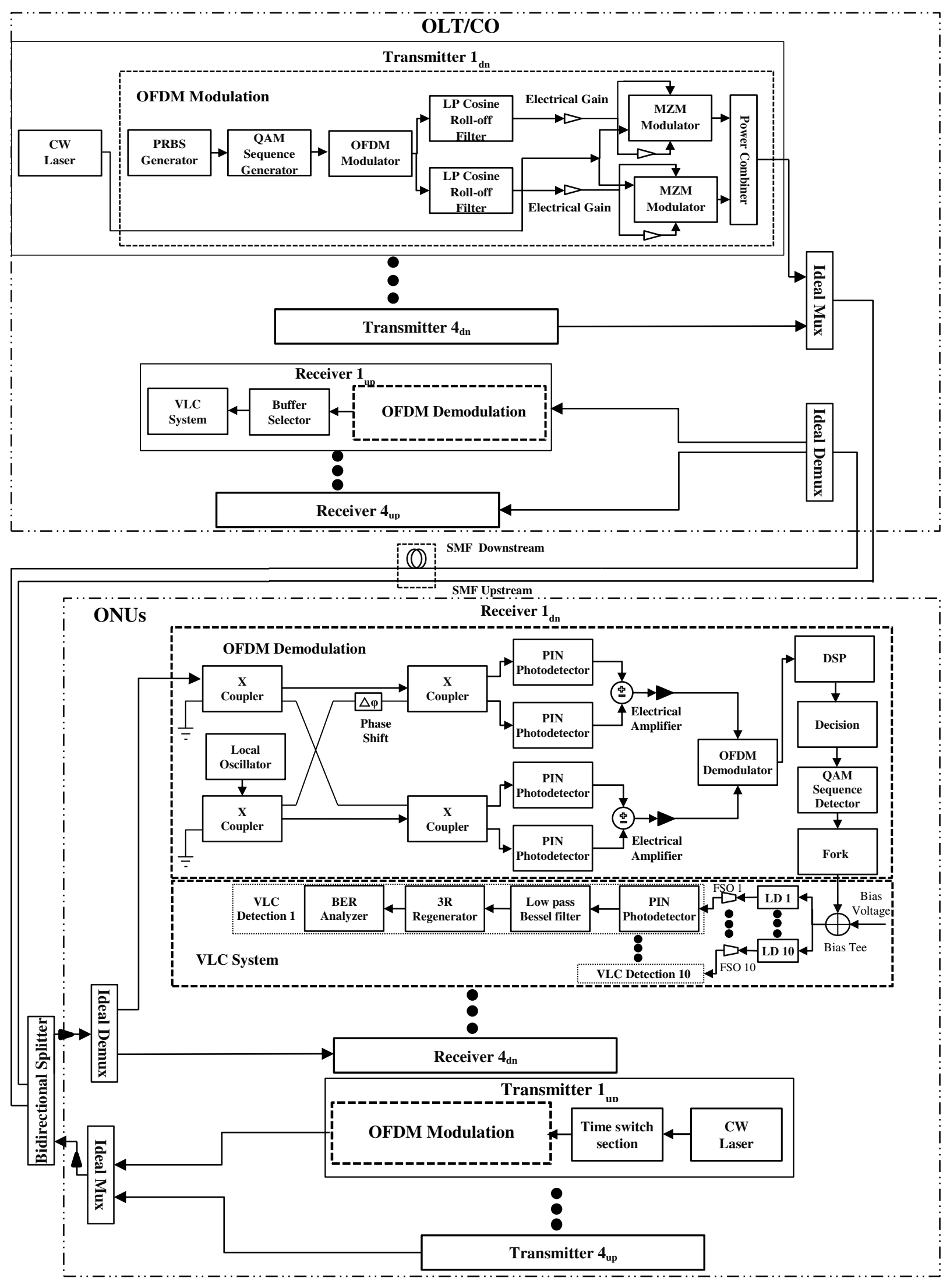

(a) 


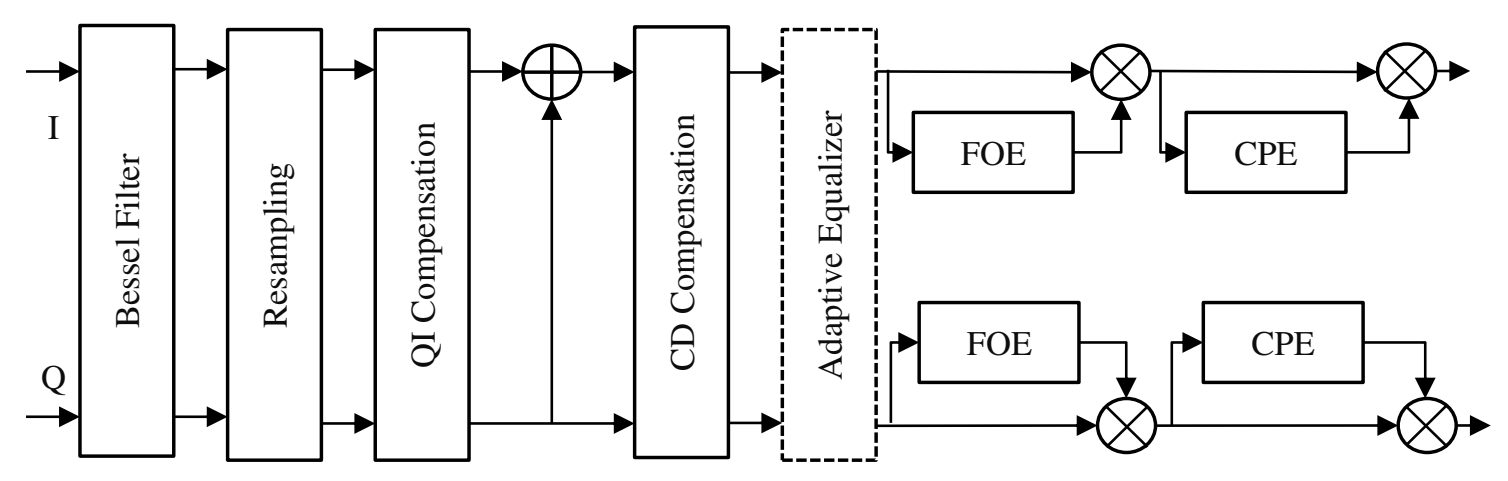

(b)

Fig. 3 Schematic diagram of (a) bidirectional four wavelengths 10/10 Gbps TWDM-PON utilizing m-QAM OFDM modulation with multi LDs based VLC system and (b) single-polarization DSP unit

The schematic diagram of a full-duplex 10/10 Gbps TWDM PON system with m-QAM-OFDM modulation over SMF for wired and free space optics (FSO) link i.e. VLC wireless transmission, is presented in Fig. 3 using OptiSystem 17.0 software. In this system, for system design, the global parameters are no. of samples $(=32768)$ and symbol rate $\left(=5 \times 10^{9} \mathrm{symbols} / \mathrm{sec}\right)$. For bidirectional transmission, both OLT and ONUs consists of four pairs of transmitters/receivers. Also, the downstream wavelengths $(1596-1598.4 \mathrm{~nm})$ and the upstream wavelengths $(1532.6-1535 \mathrm{~nm})$ follow the ITU-T channel spacing of $0.8 \mathrm{~nm}(100 \mathrm{GHz})$. The information is emitted at these frequencies by utilizing four pairs of continuous wave $(\mathrm{CW}$ ) lasers (linewidth $=0.15 \mathrm{MHz}$ and input power=-6dBm). A single downstream transmitter is illustrated in Fig. 3(a) which comprises a pseudorandom bit sequence (PRBS) generator offering the random binary sequences at 10Gbps which are fed to $\mathrm{m}$ QAM where $\mathrm{m}=16-$-, 32- and 64 with 4-, 5- and 6-bits per symbol sequence generator respectively. The m-QAM signals output is transmitted to an OFDM modulator for mapping over 512 subcarriers, through the serial to parallel converted. 512 subcarriers are employed with a fast Fourier transform (FFT) having 1024 points and a null cyclic prefix (Kaur et al. 2017b).

An OFDM modulator provides the in-phase (I) as well as quadrature (Q) components of the incoming signal for the data transmission in I/Q optical modulator and to filtered by two low pass cosine roll-off filters (roll-off factor=0.2) to forward for signal modulation. The I/Q optical modulator consists a laser signal, two electrical gain components, two mach-Zehnder modulators (MZMs) (extinction ratio=60 dB) followed by an optical power combiner ( size $=2 \times 1$ ) (Kachhatiya and Prince 2016). Further, four downstream OFDM signals are multiplexed at disctinct wavelelengths for transmission over an optical fiber. Then, the signal is forward to a splitter, an OA (gain $=13 \mathrm{~dB}$ and noise figure $=4 \mathrm{~dB}$ ) to improve the receiver's receiver sensitivity and transferred to the receiver section where to demodulate the TWDM-OFDM signals an opposite operation is accomplished. For signal reception (at ONU side), a de-multiplexed single user signal is passed to the four number of of $\mathrm{X}$ couplers (coupling coefficient $=0.5$ ) in addition to a local oscillator and phase shifter (phase shift $=90^{\circ}$ ) for coherent orthogonal reception. Again the signal is fed to the four PIN photodetectors (PDs) (responsivity =1 $\mathrm{A} / \mathrm{W}$, dark current=10 $\mathrm{nA}$ and thermal noise $=1 \times 10^{-22} \mathrm{~W} / \mathrm{Hz}$ ) followed by two electrical subtractors, two electrical amplifiers (gain=20 dB) and OFDM demodulator. After coherent OFDM demodulation, a single polarization digital signal processing (DSP) unit executes necessary functions to recovere the received signals and for nonlinearities losses compensation as shown in Fig. 3(b). In DSP unit, bessel filter is utilized to filter the required signal having specific samples/symbol $(=8 \times$ samples per bit $)$ and bandwidth $(=0.75 \times$ symbol rate $)$ whereas resampling is utilized at rate $=4$ samples/symbol. The rest of the DSP sub-unit are explained as follows (Yang et al. 2013):

IQ compensation is utilized to minimise the phase along with amplitude imbalances within respective I and Q received signals. Mathematically, for coherent receiver including DSP component, the $90^{0}$ phase shift among I and Q components is produced by $90^{\circ}$ optical and hybrid and $180^{\circ}$ phase shift between balanced detection. The optical hybrid output $E_{1}, E_{2}, E_{3}$ and $E_{4}$ by neglecting imbalance and loss is given as (Guerreiro et al. 2020):

$$
\begin{aligned}
& E_{1}=\frac{1}{\sqrt{2}}\left[E_{r}+E_{o}\right] \\
& E_{2}=\frac{1}{\sqrt{2}}\left[E_{r}-E_{o}\right]
\end{aligned}
$$




$$
\begin{aligned}
& E_{3}=\frac{1}{\sqrt{2}}\left[E_{r}-j E_{o}\right] \\
& E_{4}=\frac{1}{\sqrt{2}}\left[E_{r}+j E_{o}\right]
\end{aligned}
$$

where $E_{r}$ and $E_{o}$ depicts the received optical and local oscillator input signals. CD compensation (through BackPropagation algorithm) is utilized to eliminate $\mathrm{CD}$ as well as fiber nonlinearity. Also, photocurrent i.e. $I_{1}$ and $I_{2}$ from two photodetectors at coherent receiver are given as (Guerreiro et al. 2020):

and

$$
I_{1}=\frac{1}{2}\left\{\left|E_{r}\right|^{2}+\left|E_{o}\right|^{2}+2 \operatorname{Re}\left[E_{r} E_{o}^{*}\right]\right\}
$$

$$
I_{2}=\frac{1}{2}\left\{\left|E_{r}\right|^{2}+\left|E_{o}\right|^{2}-2 \operatorname{Re}\left[E_{r} E_{o}^{*}\right]\right\}
$$

Thus, the ultimate complex detected signal comprising I and Q phase components are presented as (Guerreiro et al. 2020):

$$
I(t)=I_{I}(t)+j I_{Q}(t)=2 E_{r} E_{o}^{*}
$$

After this, to de-multiplex the polarization of received signal, an adaptive equalizer offers a butterfly structure. Frequency offset estimation (FOE) is employed to reduce the phase as well as frequency unmatched between the local oscillator at receiver side and transmitter. Further, the signal obtained at the input single polarization DSP unit, $R(k)$ with data symbol, $I(k)$ and zero-mean Gaussian random variable, $N(k)$ is expressed as (Yang et al. 2013):

$$
R(k)=I(k) \cdot e^{j\left(2 \pi \Delta f k T+\varphi_{k}\right)}+N(k)
$$

where $\varphi_{k}, \Delta f$ and $T$ depict carrier phase, carrier frequency offset and symbol period respectively. Again, the fourth power $R(k)$ can be expressed as (Yang et al. 2013)

$$
R^{4}(k)=C \cdot e^{4 j\left(2 \pi \Delta f k T+\varphi_{k}\right)}+Z(k)
$$

where $C$ is the constant amplitude, $Z(k)$ mean noise process together with zero-mean. Also, taking into account the signal's spectral density, the frequency offset can be represented as (Yang et al. 2013):

$$
R^{4}(k)=\frac{1}{4} \arg \{\max [|G(f)|]\}
$$

where

$$
|G(f)|=\frac{1}{n} \sum_{k=0}^{n-1} C^{4}(k) \cdot e^{-j(2 \pi \Delta f k T)}
$$

where $n$ means block length. Then, carrier phase estimation (CPE) is utilized to offer optimum output in terms of distance and information rate. In $2 \mathrm{D}$ complex plane, the estimated required space $\left|s_{k, b}\right|^{2}$ to the adjacent constellation point by decision component is expressed as (Yang et al. 2013):

$$
\left|s_{k, b}\right|^{2}=\left|G_{k} e^{-j \varphi_{b}}-X_{k, b}\right|^{2}
$$

where $X_{k, b}$ denotes the decision $G_{k} e^{-j \varphi_{b}}$. At last for polarization, the decoding of received sequences, parallel to serial conversion processed to acquire the final output bits.

After the DSP unit, a decision component followed by an m-QAM sequence generator is used for coherent downlink traffic detection. OFDM demodulator performance parameters should be identical with the transmitter to recover the m-QAM symbols at receiver side. The m-QAM sequence detector signifies the binary sequences and de-mapped the received electrical signal to retrieve the bit sequences (Kachhatiya and Prince 2018; Kaur et al. 2017a). Then for wireless transmission, a $1 \times 10$ fork is used for copying the incoming signal into ten numbers of ideal single-mode LDs $(390,430,470,510,550,590,630,670,710$ and $750 \mathrm{~nm})$ based 
VLC system. Here, each ideal single-mode LD provides no internal cavity losses over the visible light range $(375-780 \mathrm{~nm})$. Further, to drive each LD (input power=5 $\mathrm{dBm}$ and modulation peak current $=9 \mathrm{~mA}$ ), the DC bias signal $(4 \mathrm{~V})$ and incoming electrical signals are integrated using a bias tee and transmitted via the FSO link. Each VLC system consists of ten numbers of FSO links corresponding to ten numbers of multi-color LDs for high speed and long-range point-to-point communication. Then, the received signal is detected using PIN PD followed by a low pass Bessel filter (LPF) (cut-off frequency $=3.75 \mathrm{THz}$ ), $3 \mathrm{R}$ regenerator for recovering the received signal and bit error rate (BER) analyzer to obtain the output.

Considerably, for uplink direction, $4 \times 10$ Gbps m-QAM OFDM signals are produced with four upstream TWDM wavelengths (1532.6-1535 nm). The uplink 40 Gbps traffic transmitted from the ONUs and detected at $\mathrm{CO}$ side. Also, these independent upstream wavelengths are transmitted by using CW lasers and each $\mathrm{CW}$ laser wavelength is passed through a time switch section for upstream transmission as shown in Fig. 4.

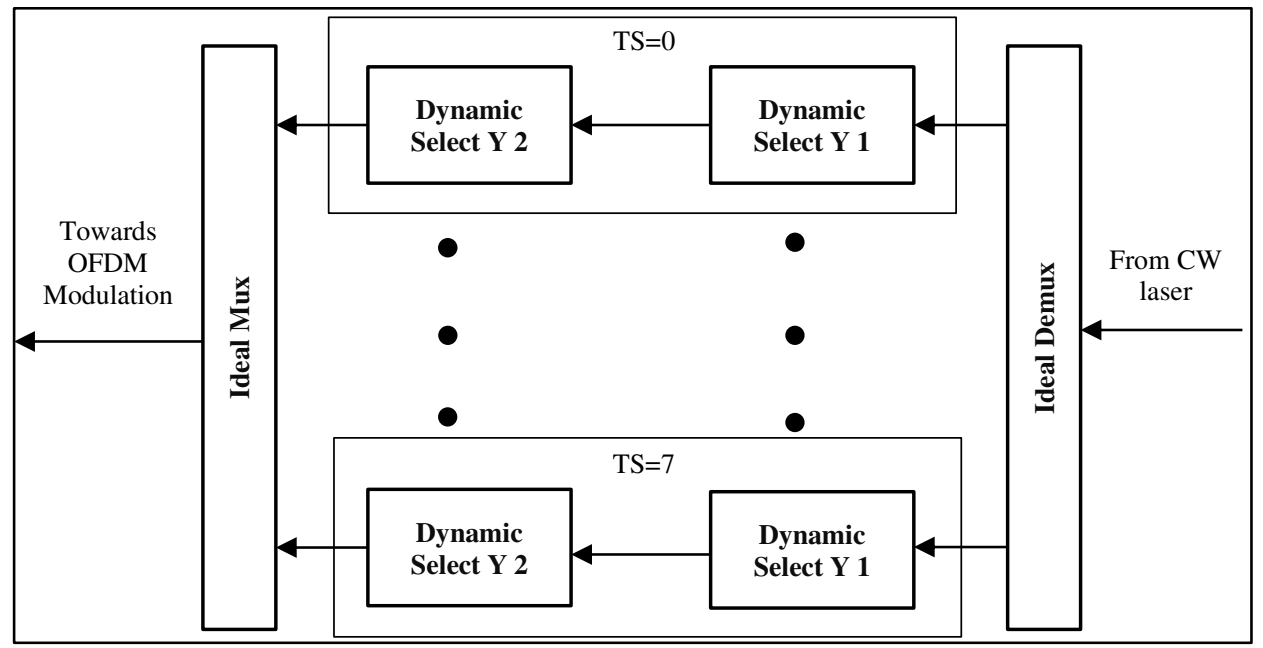

Fig. 4 Upstream time switch section

Eight pairs of two cascaded dynamic selects Y per wavelength, to transmit the information at definite timeslot (TS) and switching time ( $\mathrm{Ts}_{1}$ and $\mathrm{Ts}_{2}$ ) are used for the upstream time switch section as (Kumari et al. 2019):

and

$$
\mathrm{Ts}_{1}=\mathrm{TS} \cdot\left(\frac{1}{\text { Bit rate }}\right) \cdot\left(\frac{\mathrm{SL}}{\mathrm{T}}\right)
$$

$$
\mathrm{Ts}_{2}=\mathrm{Ts}_{1}+\left(\frac{\mathrm{TW}}{\mathrm{T}}\right)
$$

where TS (=0 to 7), SL (=8192), T (=8) and TW $\left(=0.81 \times 10^{-06} \mathrm{~s}\right)$ denote the timeslot, sequence length, number of customers utilizing the same upstream wavelength and time window respectively at a reference wavelength of $1550 \mathrm{~nm}$. Table 1 shows the switching time of the proposed system.

Table 1 Switching Time for the upstream transmission at $10 \mathrm{Gbps}$

\begin{tabular}{|c|c|c|}
\hline Timeslot & $\mathbf{T}_{\mathbf{s 1}}(\mathbf{s})$ & $\mathbf{T}_{\mathbf{s} 2}(\mathbf{s})$ \\
\hline 0 & 0 & $0.10 \times 10^{-06}$ \\
\hline 1 & $0.10 \times 10^{-06}$ & $0.20 \times 10^{-06}$ \\
\hline 2 & $0.20 \times 10^{-06}$ & $0.30 \times 10^{-06}$ \\
\hline 3 & $0.30 \times 10^{-06}$ & $0.40 \times 10^{-06}$ \\
\hline 4 & $0.40 \times 10^{-06}$ & $0.51 \times 10^{-06}$ \\
\hline 5 & $0.51 \times 10^{-06}$ & $0.61 \times 10^{-06}$ \\
\hline 6 & $0.61 \times 10^{-06}$ & $0.71 \times 10^{-06}$ \\
\hline 7 & $0.71 \times 10^{-06}$ & $0.81 \times 10^{-06}$ \\
\hline
\end{tabular}

In the time switch section, an ideal multiplexer is employed to deliver the OFDM modulation switching information in upstream direction and passed through an OFDM modulator and an OA. Then each uplink wavelength is detected at the OLT side employing an OFDM demodulator. To choose the recent simulation iteration a buffer selector is utilized. Then the received signal is transferred towards a multi-color LDs based 
VLC system for uplink wireless communication. In ODN section, two identical SMF links using a bidirectional splitter are employed to isolate the uplink and downlink wavelengths in the ONUs side. Table 2 presents the parameters utilized in this proposed work.

Table 2 Proposed work SMF/VLC link parameters

\begin{tabular}{|c|c|c|c|c|}
\hline Component Name & \multicolumn{2}{|c|}{ Parameters } & Value & Unit \\
\hline \multirow{8}{*}{ SMF } & \multicolumn{2}{|c|}{ Wavelength (reference) } & 1550 & $\mathrm{~nm}$ \\
\hline & \multicolumn{2}{|c|}{ Length } & $10-50$ & $\mathrm{~km}$ \\
\hline & \multicolumn{2}{|c|}{ Attenuation } & 0.2 & $\mathrm{~dB} / \mathrm{km}$ \\
\hline & \multicolumn{2}{|l|}{ Dispersion } & 16.75 & $\mathrm{ps} / \mathrm{nm} / \mathrm{km}$ \\
\hline & \multicolumn{2}{|c|}{ Dispersion slope } & 0.075 & $\mathrm{ps} / \mathrm{nm}^{2} / \mathrm{k}$ \\
\hline & \multicolumn{2}{|c|}{ Effective area } & 80 & $\mu \mathrm{m}^{2}$ \\
\hline & \multicolumn{2}{|c|}{$\begin{array}{l}\text { Nonlinear index of } \\
\text { refraction }\end{array}$} & $26 \times 10^{-21}$ & $\mathrm{~m}^{2} / \mathrm{W}$ \\
\hline & \multicolumn{2}{|c|}{ Temperature } & 300 & $\mathrm{~K}$ \\
\hline \multirow{5}{*}{ FSO } & \multicolumn{2}{|l|}{ Range } & $10-8000$ & $\mathrm{~m}$ \\
\hline & \multicolumn{2}{|c|}{ Attenuation } & 8 & $\mathrm{~dB} / \mathrm{km}$ \\
\hline & \multirow{2}{*}{$\begin{array}{l}\text { Aperture } \\
\text { diameter }\end{array}$} & Transmitter & 5 & $\mathrm{~cm}$ \\
\hline & & Receiver & 10 & $\mathrm{~cm}$ \\
\hline & \multicolumn{2}{|c|}{ Beam divergence angle } & 1108 & $\operatorname{mrad}$ \\
\hline
\end{tabular}

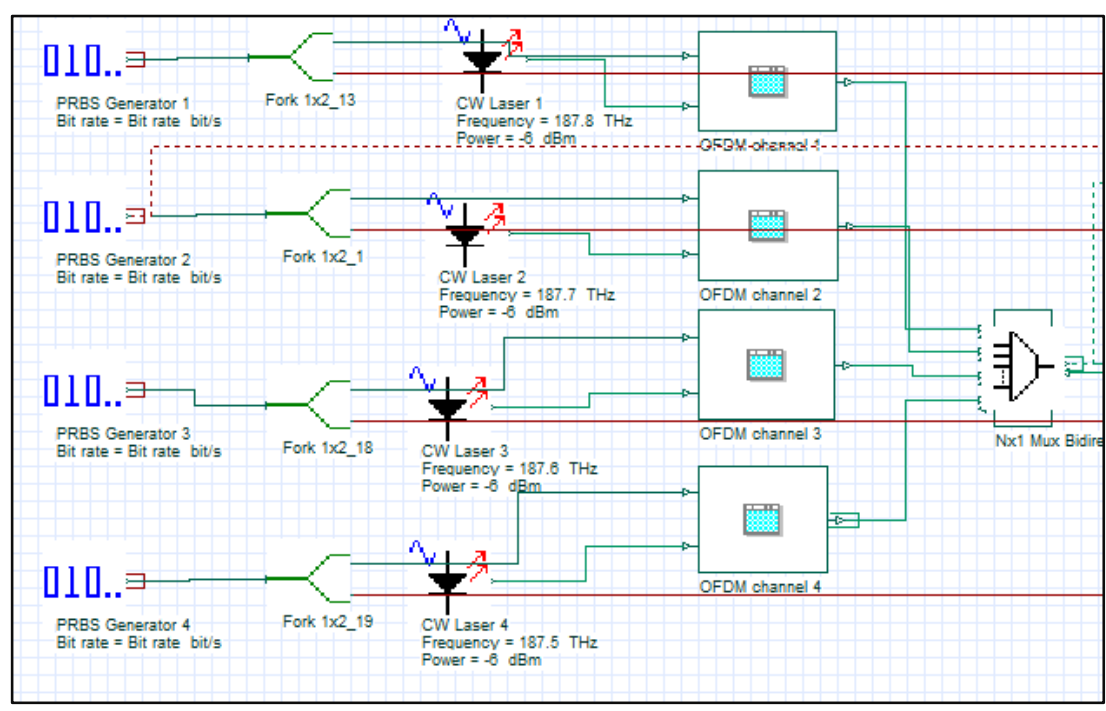

(a) Downstream transmitter 


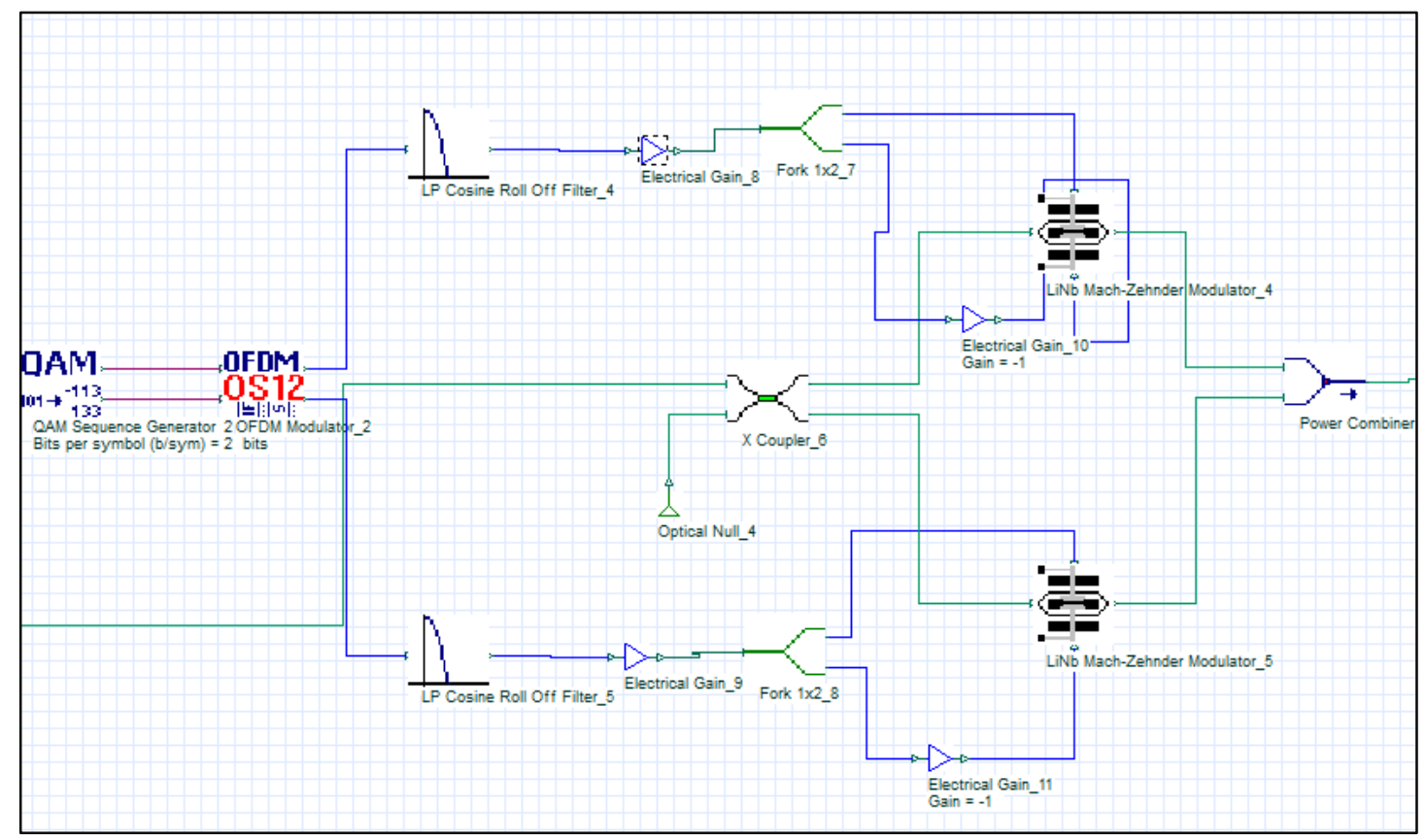

(b) OFDM channel

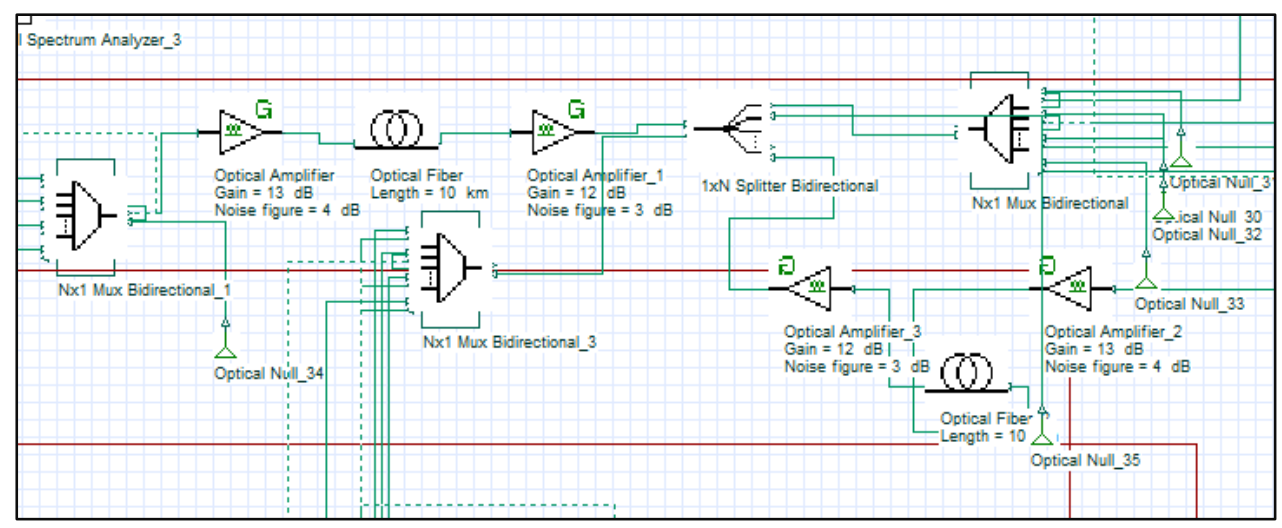

(c) Bidirectional channel

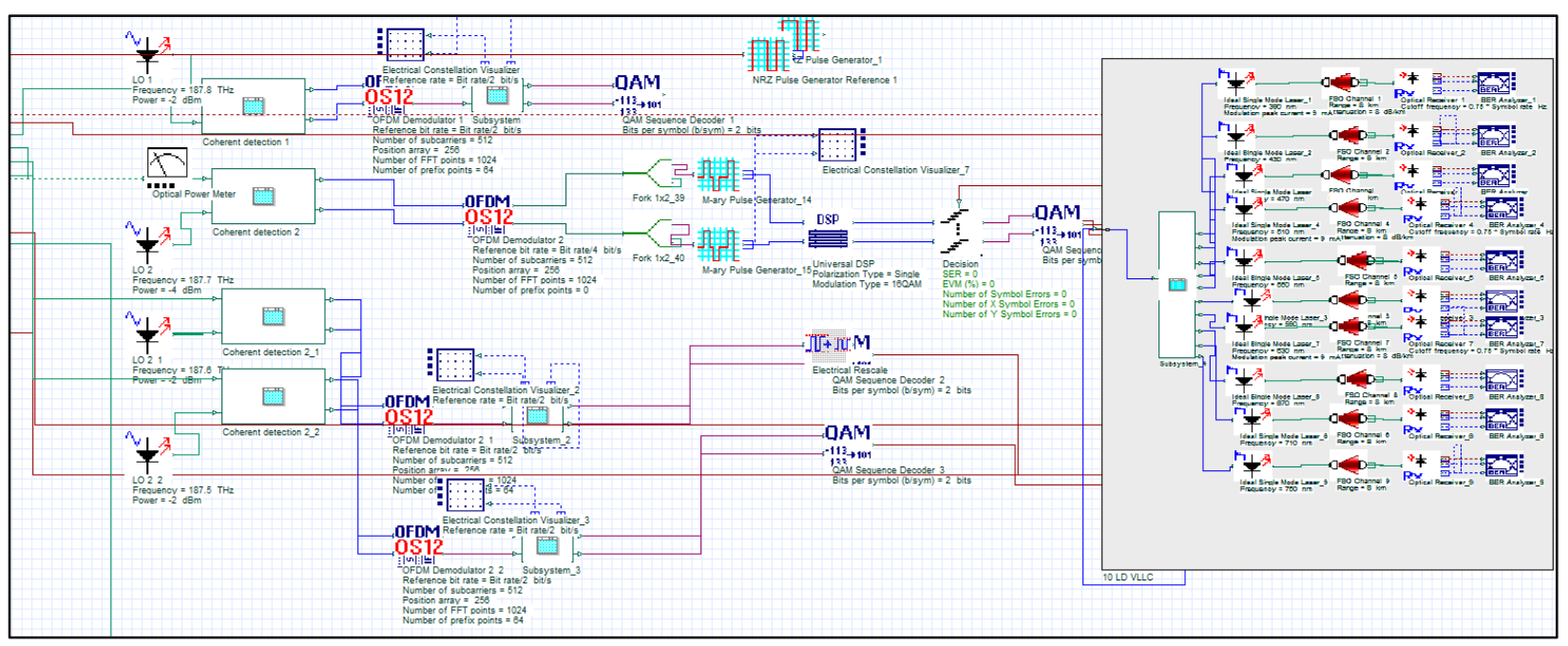

(d) Downstream Receiver 


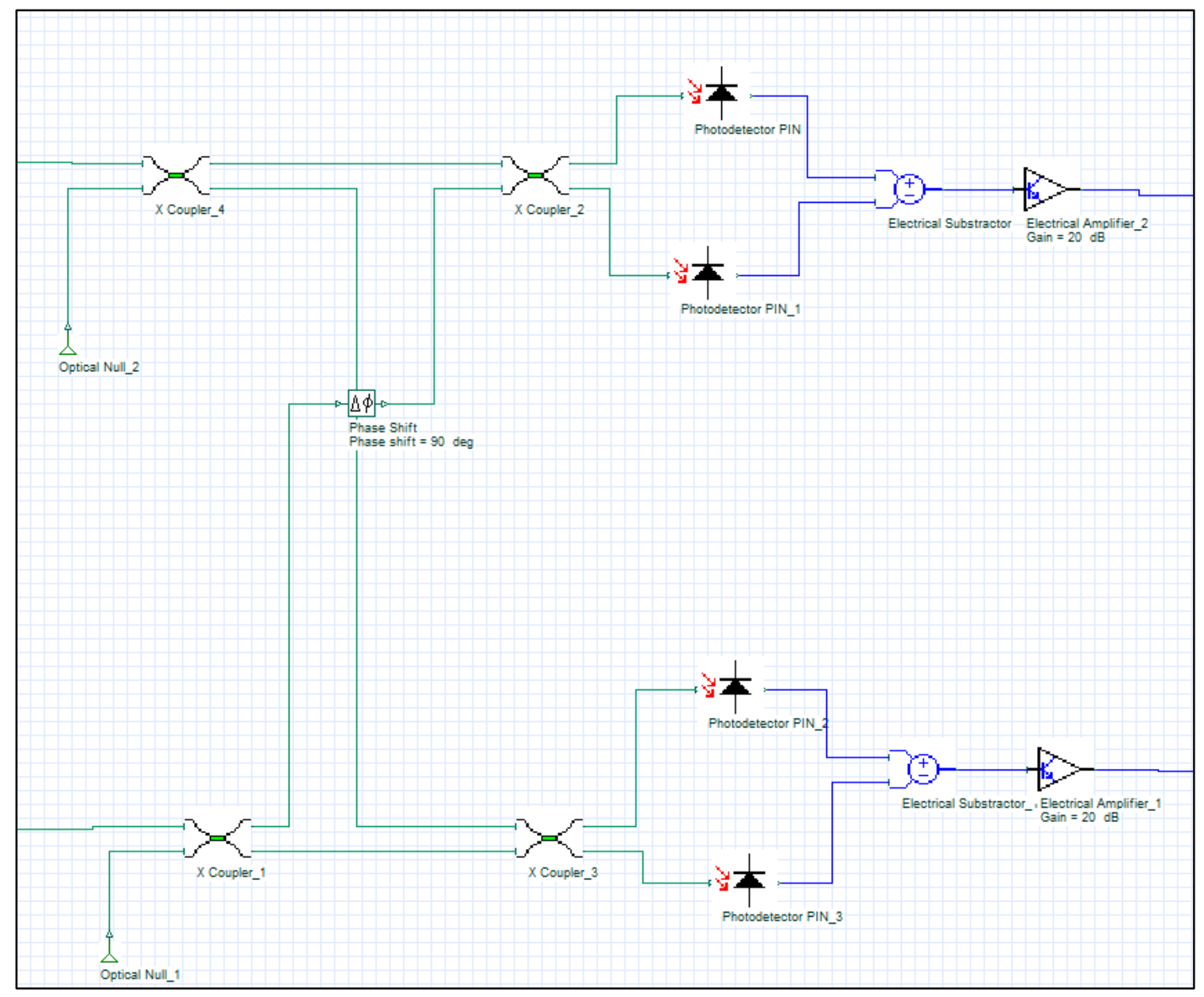

(e) Coherent detection

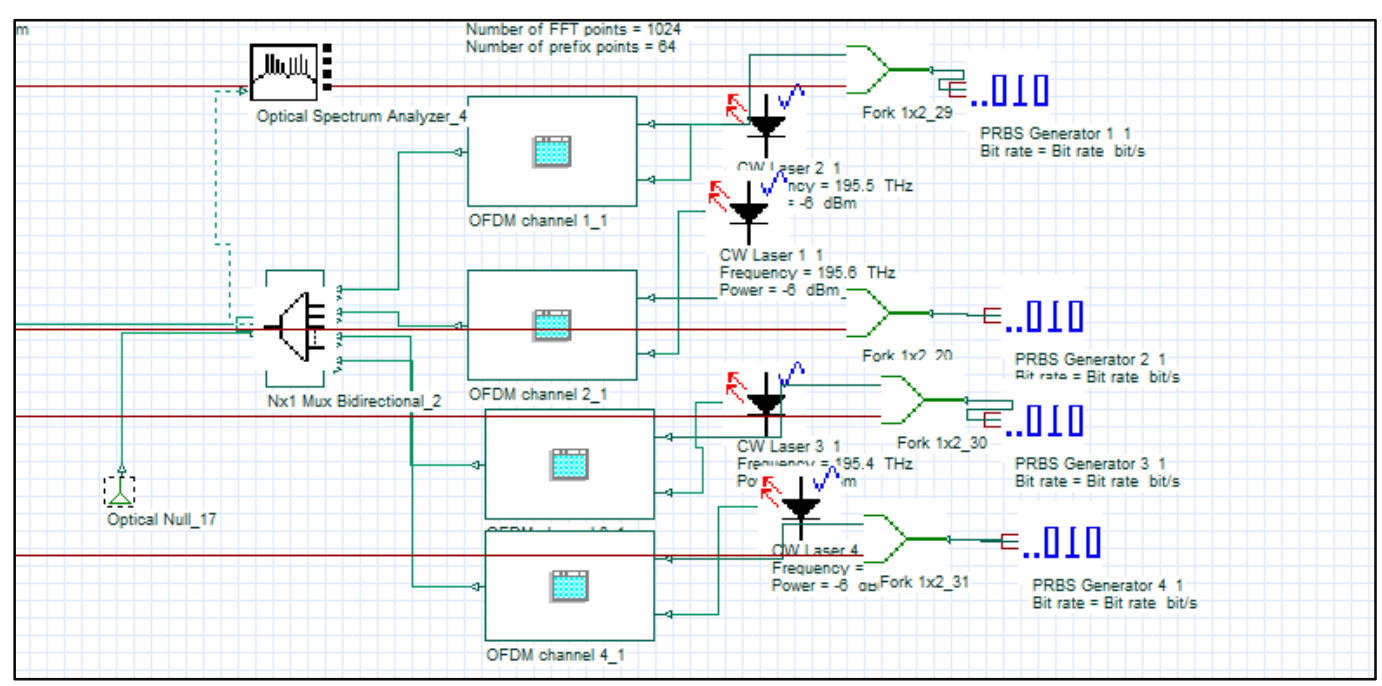

(f) Upstream transmitter 


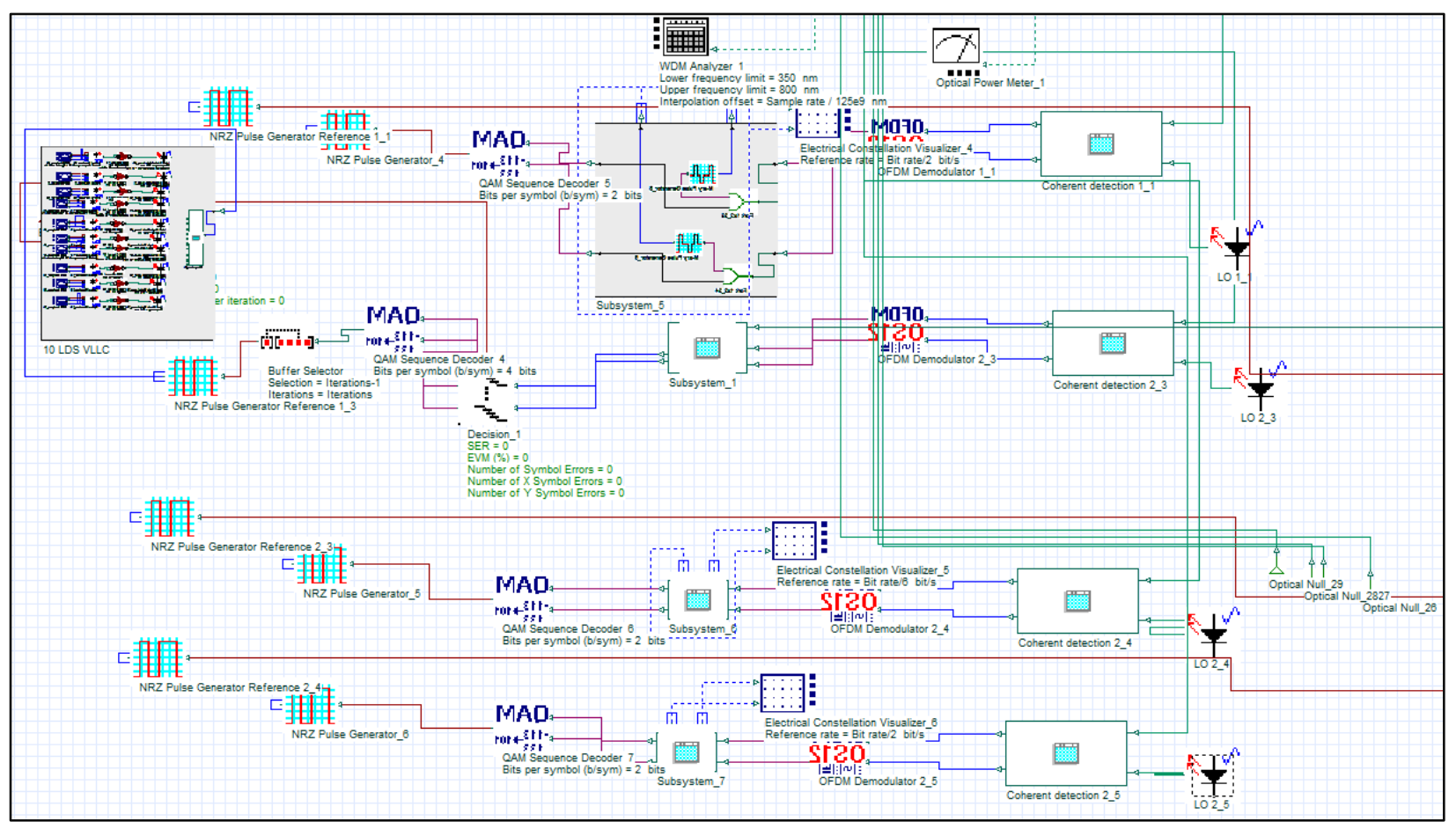

(g) Upstream receiver

Fig. 5 Simulation diagram of full-duplex $4 \times 10$ Gbps TWDM-PON using m-QAM OFDM modulation with VLC system (a) downstream transmitter, (b) OFDM channel, (c) bidirectional channel, (d) downstream Receiver, (e) coherent detection, (f) upstream transmitter and (g) upstream receiver

The schematic designed layout for the TWDM PON system with 16-, 32- and 64-QAM-OFDM modulation using OptiSystem software is shown in Fig. 5.

\section{Numerical analysis}

In this section the basic numerical analysis of the proposed PON/VLC link including fiber impairments issues is presented to validate the feasibility of the proposed system under fiber impairments. The proposed coherent detection OFDM in TWDM PON and VLC system offers high receiver sensitivity for long reach transmission. As in coherent OFDM the large number of subcarriers can cause the channels interference and hence degrades the system performance while using the too small polarization results in reduction in the spectrum utilization. In general without using DSP component, the $\mathrm{CD}, D$ in the proposed fiber/VLC link, $l$ is presented using the education $D=l[d+s(\lambda-1550)]$ in ps.nm where $\lambda, d$ and $s$ are wavelength, dispersion coefficient and slope coefficient respectively at reference wavelength of $1550 \mathrm{~nm}$. For $10 \mathrm{~km}$ fiber and $10 \mathrm{~m}$ of VLC range at data rate of $10 \mathrm{Gbps}$ the calculated $D$ is approximate $205 \mathrm{ps.nm}$ and for $50 \mathrm{~km}$ and $10 \mathrm{~m}$ of fiber and VLC ranges respectively at same data rate is calculated as $1020 \mathrm{ps} . \mathrm{nm}$ which is below than defined tolerance of $1176 \mathrm{ps} / \mathrm{nm}$ at transmission rate of $10 \mathrm{Gbsp}$ per wavelength. But with increase in transmission distance and transmission rate the, the value for $D$ increases and hence performance of the system decreases. Also, at higher transmission rate causes spreading of optical pulses or inter-symbol interference (ISI) (Kumari et al. 2020; Mandal et al. 2021).

Again, for the proposed fiber/VLC link the pulse broadening, $\Delta t$ using the expression $\Delta t=d l \Delta \lambda$ in ps where $\Delta \lambda$ means spectral line width is $84 \mathrm{ps}$ over $50 \mathrm{~km}$ wired and $10 \mathrm{~m}$ VLC link ranges at 10 Gbap data rate. In addition, the calculated unwanted side bands i.e. four wave mixing (FWM) wavelengths $\mathrm{W}$, for $\mathrm{N}$ channels with 0.8 channel spacing in fiber link is approx. 21 using the expression is $W=\frac{N^{2}}{2(N-1)}$. Hence, there is need to control the subcarriers in coherent OFDM. In addition, DSP unit can also affect the performance of system in terms of long reach distance and high speed transmission capacity by minimizing fiber impairments such dispersion, non-linear effects and polarization losses. The impact of fiber nonlinearities because of the fiber transmission along with their mitigation utilizing the proposed CO-OFDM with DSP unit receiver are illustrated as follows (Kumari et al. 2020; Mandal et al. 2021) :

A polarization independent phenomenon i.e. chromatic dispersion (CD) can be compensated before demultiplexing as well as equalizing the received signal to retrieve the two orthogonal polarization channels at transmitter side. The expression for CD can be expressed as (Kumari et al. 2020; Renaudier et al. 2010): 


$$
D(x, \varphi)=\exp \left(-i \frac{d \lambda^{2}}{4 \pi c} \varphi^{2}\right)
$$

where $x$ and $\varphi$ are transmission distance and angular frequency respectively. $i, d, \lambda$ and $c$ are imaginary part, dispersion coefficient, wavelength and speed of light respectively. Practically by employing finite impulse response (FIR) filters of DSP unit, CD can be compensated and CD compensation amount depends on calculation capacity of state of knowledge technologies. The DSP unit's primary part is received signal demultiplexing to retrieve the orthogonal polarization transmitted at the transmitter side using FIR filters. The optical signal received at single-polarization filter structure is given as (Renaudier et al. 2010):

$$
y_{\text {out }}=h_{y} \cdot y_{\text {in }}
$$

where $y_{\text {in }}$ means input signal, $y_{\text {out }}$ presents the output signal. $h_{y}$ means the adaptive FIR filter with tap coefficient. Moreover, frequency domain equalizers are mostly used as a most popular and promising CD filters and its transfer function for coherent transmission system is represented in equation (15) (Xu 2017). After two polarization channels separation by the adaptive equalizer, phase-tracking is done in digital domain. To recover and remove the persisting phase mismatch between the signal and the local oscillator, CPE process is used (Renaudier et al. 2010). For CPE one-tap normalized LMS filter is effectively employed for coherent m-QAM transmission in the proposed system and the tap weight can be presented as (Xu 2017):

where

$$
w(n+1)=w(n)+\frac{\varepsilon}{\left|y_{\text {in }}(n)\right|^{2}} y_{i n}^{*}(n) e(n)
$$

$$
e(n)=D(n)-w(n) \cdot y_{\text {in }}(n)
$$

where $w(n+1)$ presents the tap weight; $n, D(n)$ and $e(n)$ mean symbol index, desired symbol and carrier phase estimation error between output signal and desired symbol respectively. Also, $\varepsilon$ means the step size in one-tap normalized LMS filter in the DSP unit. Thus BER in m-QAM coherent transmission system can be expressed as (Mandal et al. 2021; Xu 2017):

$$
B E R=\frac{1}{\log _{2}(m)} \operatorname{erfc}\left(\frac{\pi}{m \sqrt{2 \delta}}\right)
$$

where $\delta$ means the phase noise variance.

\section{Results and discussion}

This section describes the performance analysis of the proposed PON/VLC link for a single downstream OFDM $(1596.8 \mathrm{~nm})$ and a single upstream OFDM $(1533.4 \mathrm{~nm})$ signal where each signal consists of ten different color VLC links. Fig. 6 to 10 show optical spectra, BER and error vector magnitudes (EVM) performance of the proposed wired/wireless links in the presence of fiber distortions and noise. 


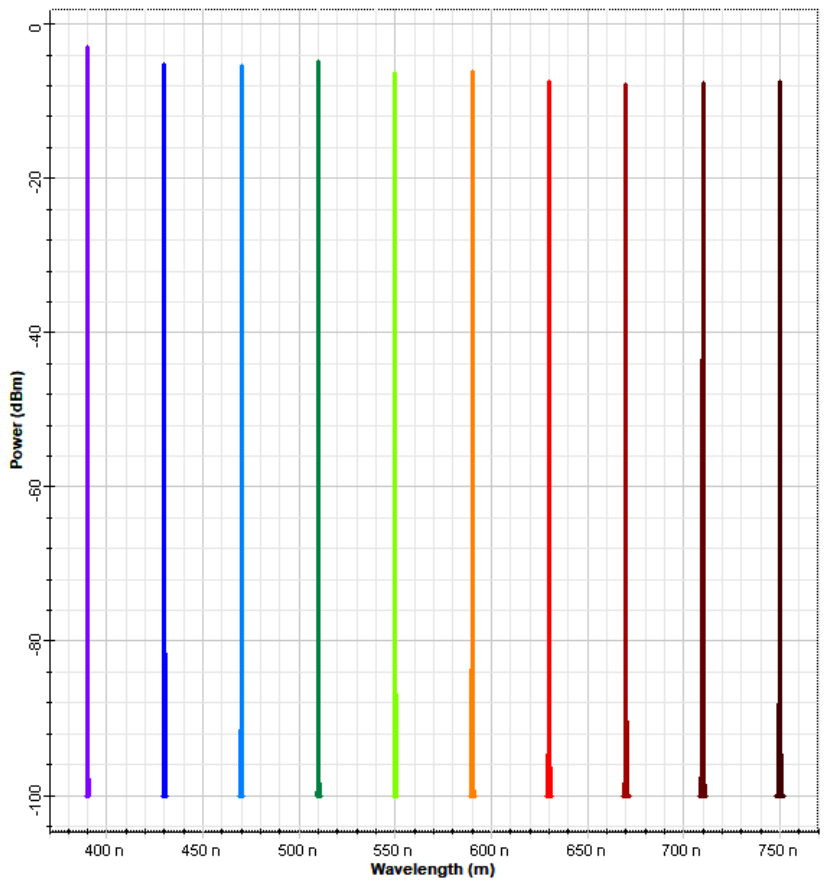

Fig. 6 The optical spectrum of multi-color LDs signals in the visible range

Fig. 6 presents the optical spectrum of the downstream multi-color LDs based VLC signals, which is obtained from an optical spectrum analyzer. It shows ten wavelengths (ten colors) from ten LDs where each LD having input power and modulation peak current of $5 \mathrm{dBm}$ and $9 \mathrm{~mA}$ respectively in the proposed system. Fig. 7 illustrates the output of the proposed PON/VLC link having $10 \mathrm{~m} \mathrm{VLC}$ and $50 \mathrm{~km} \mathrm{SMF}$ reach at $10 \mathrm{Gbps}$ data rate in downstream direction at each stage. 


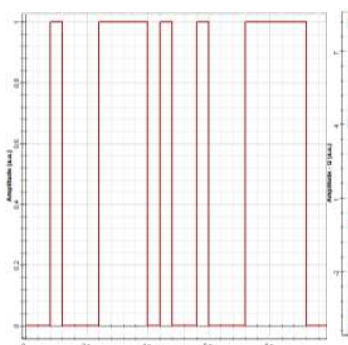

(a)

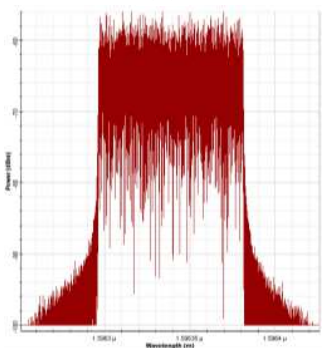

(e)

(i)

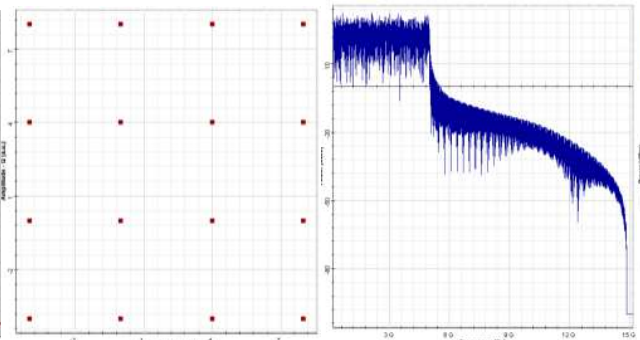

(b)

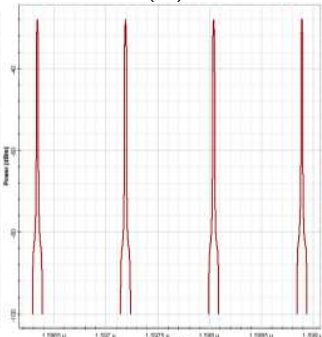

(f)

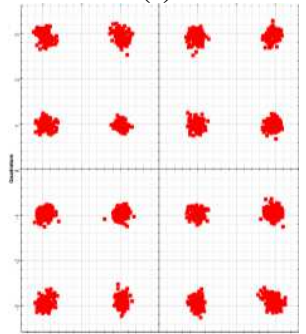

(j)

(c)

(g)

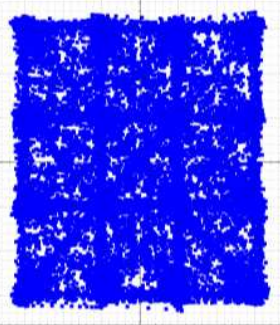

(k)
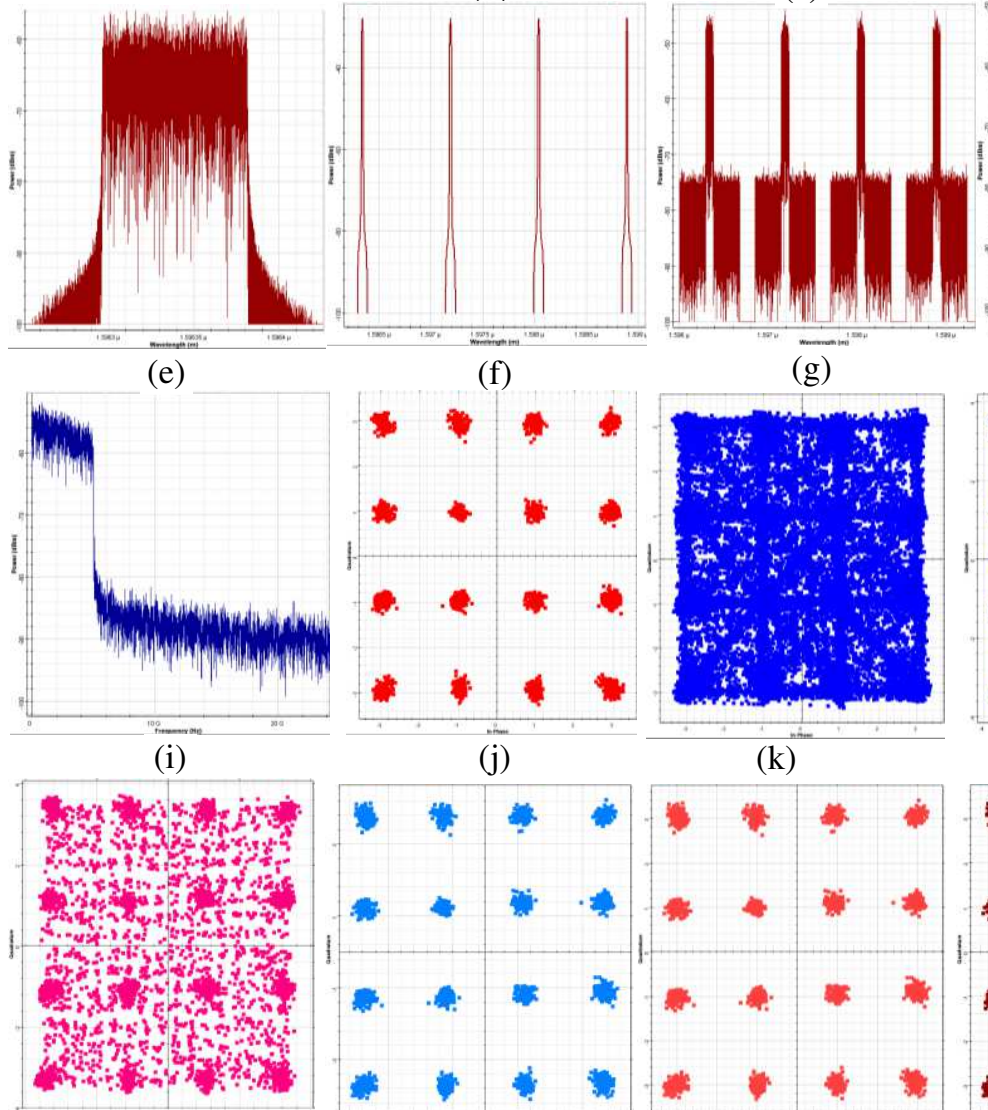

(m)

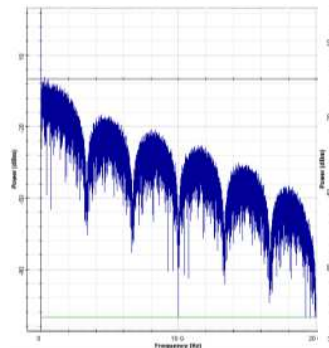

(q)

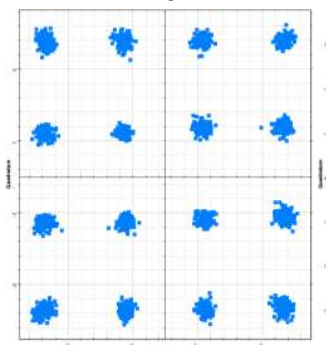

(n)

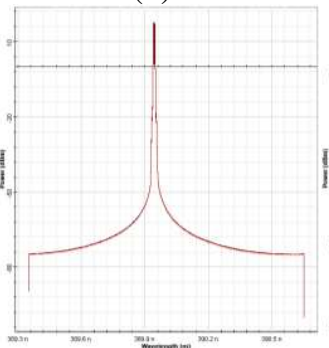

(r)

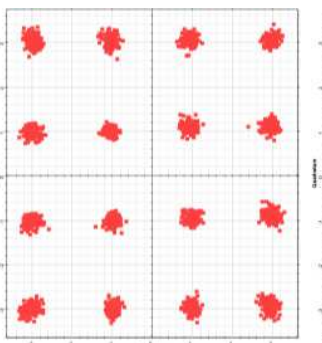

(o)

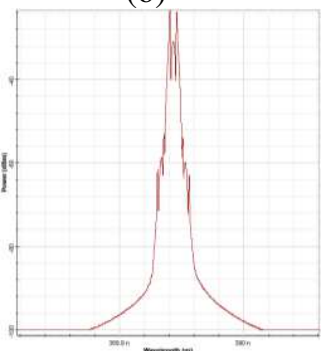

(s)

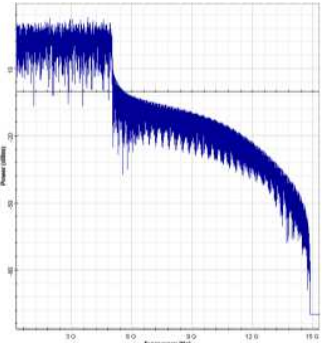

(d)

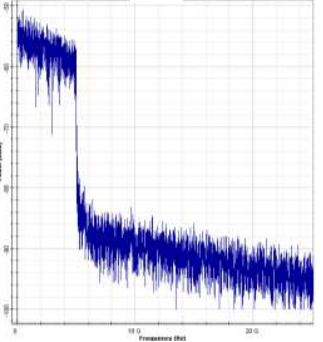

(h)

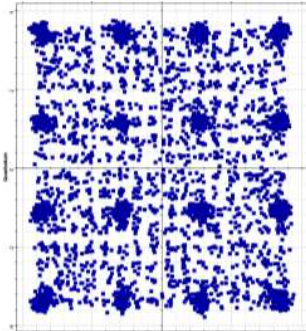

(1)
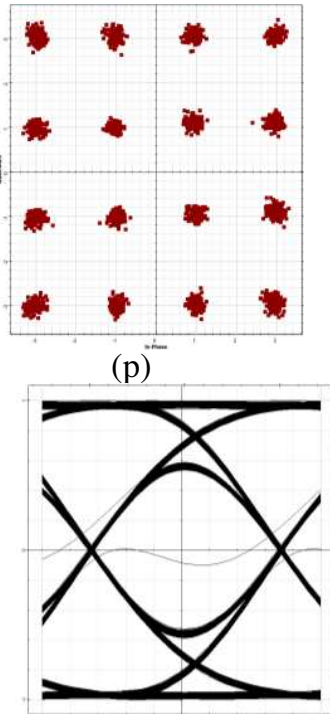

(t)

Fig. 7 Proposed system of 10/10 Gbps TWDM-OFDM PON with multi-LDs based VLC outputs at single downlink transmitter stage (a) random bit sequence; (b) 16-QAM constellation; OFDM output for (c) in-phase and (d) quadrature channels; (e) OFDM modulation output, emitted spectra of 16-QAM OFDM modulation outputs over the fiber link (f) before $50 \mathrm{~km},(\mathrm{~g})$ after $50 \mathrm{~km}$, downlink receiver stage photo detector as well as OFDM demodulated output for (h) in-phase and (i) quadrature channels; at receiver the received 16-QAM constellation diagram after $(\mathrm{j})$ OFDM demodulator, (k) bessel filtering, (l) resampling process, (m) nonlinear and dispersion compensation, (n) an adaptive equalizer, (o) output at FEO and (p) CPE, (q) signal electrical spectrum after 16-QAM, (r) output optical spectrum of LD at $390 \mathrm{~nm}$ wavelength, (s) optical spectrum after $10 \mathrm{~m}$ VLC link and (t) BER analyser eye diagram 


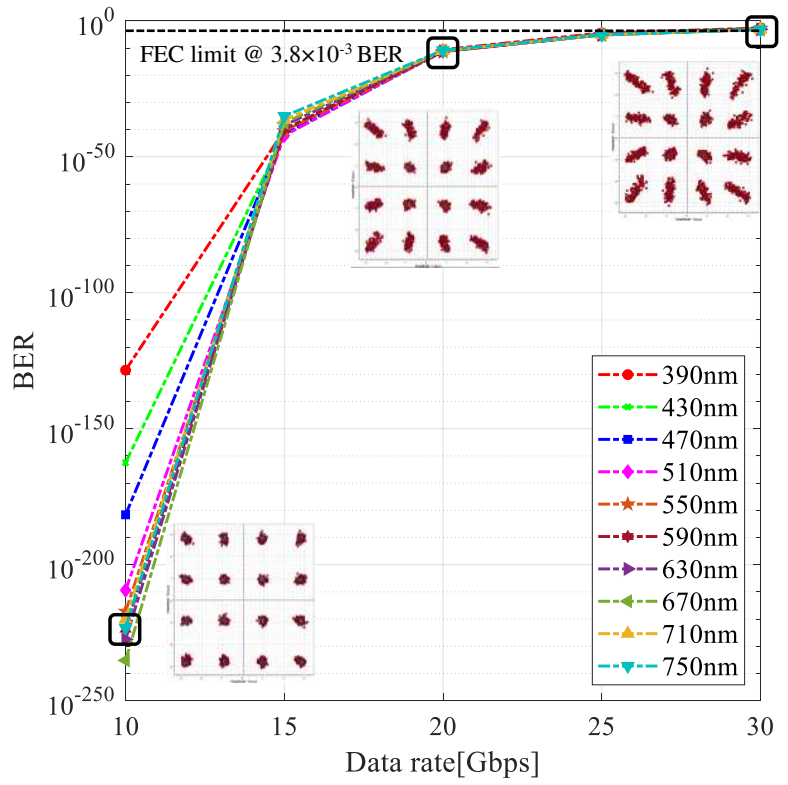

(a)

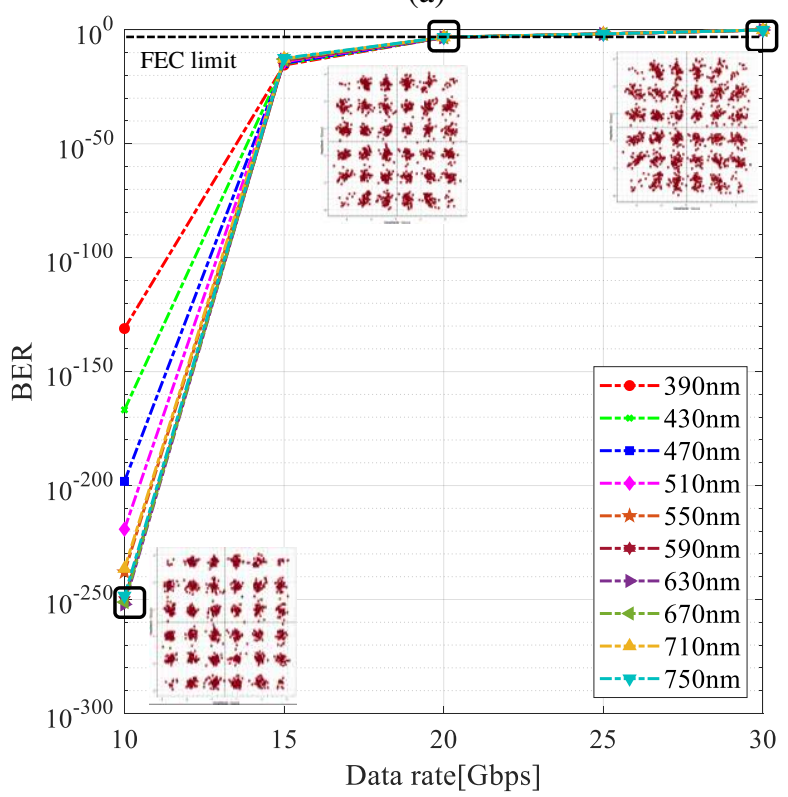

(c)

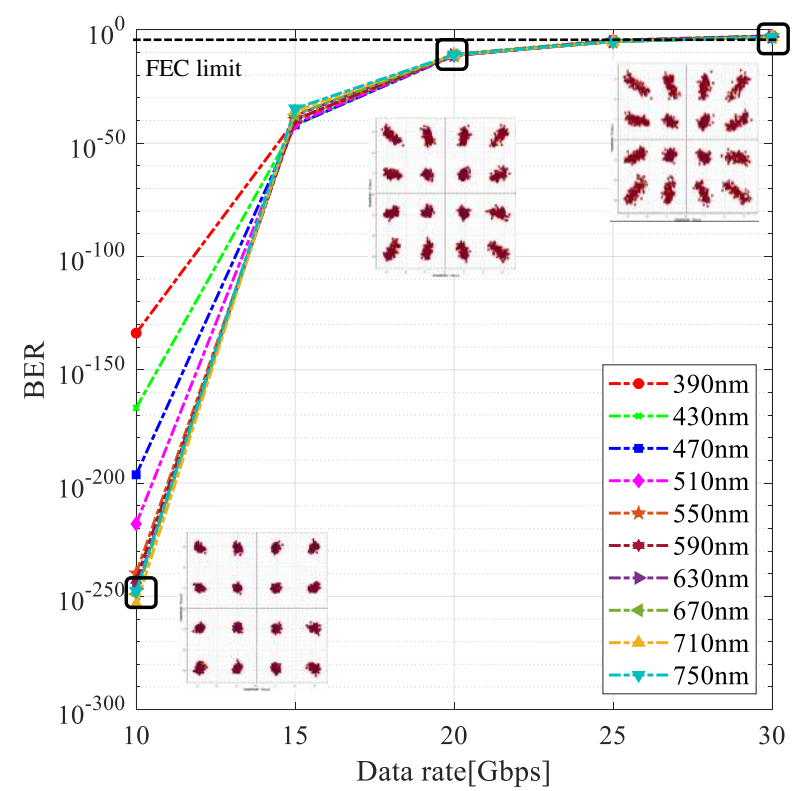

(b)

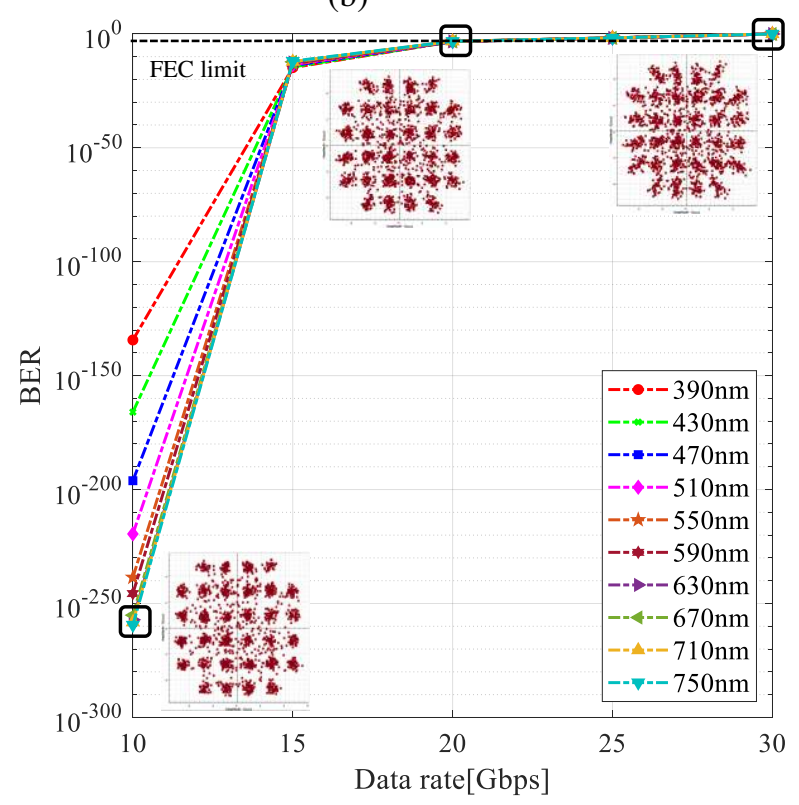

(d) 


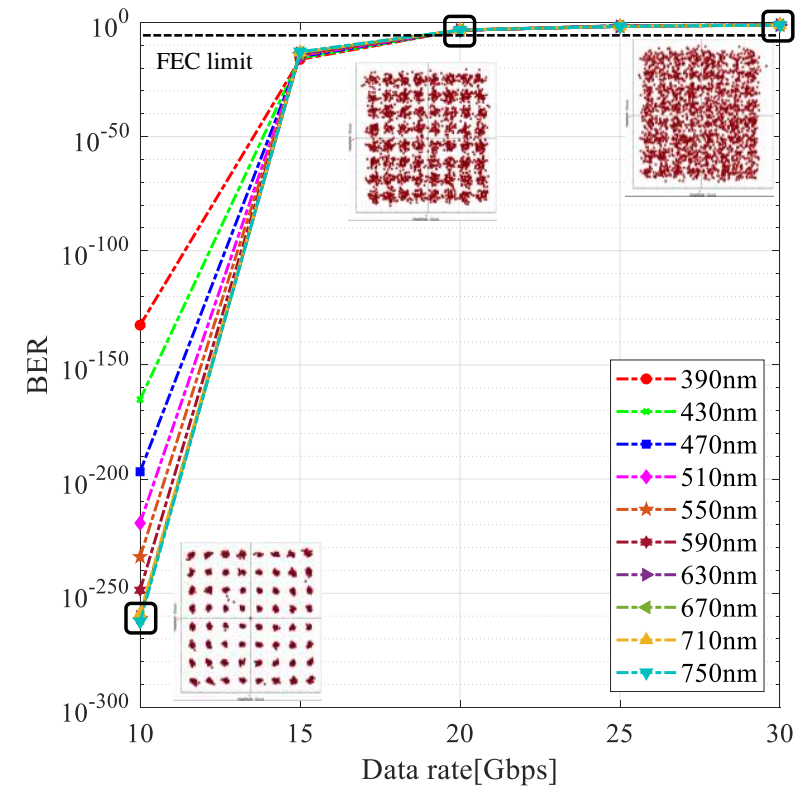

(e)

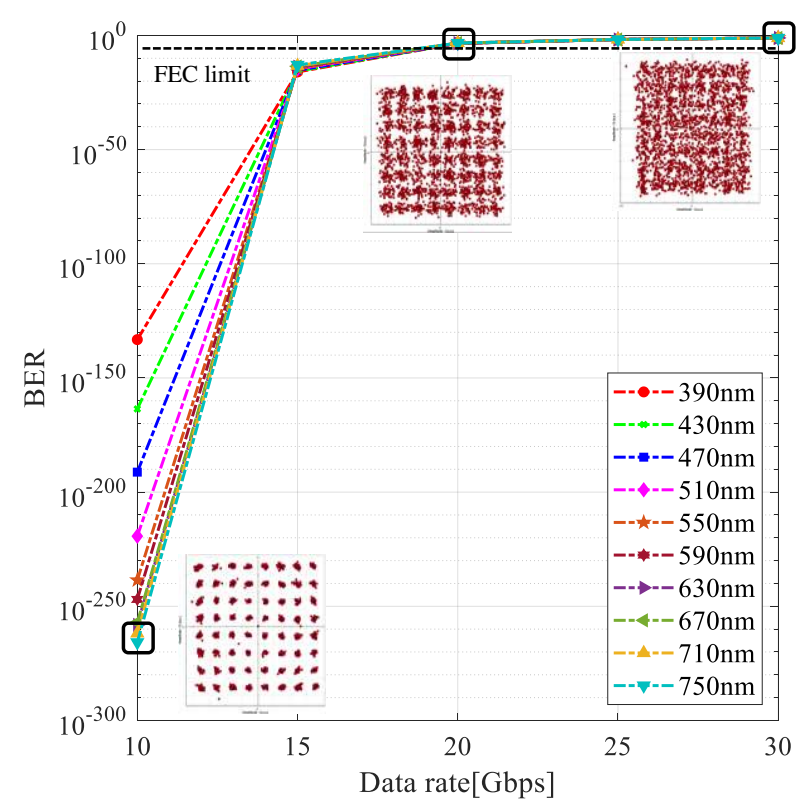

(f)

Fig. 8 BER versus data rate of different wavelengths VLC signals for (a) downstream 16-QAM, (b) upstream 16-QAM, (c) downstream 32-QAM, (d) upstream 32-QAM, (e) downstream 64-QAM and (f) upstream 64-

QAM modulation formats. Insets show the corresponding constellation diagram at different data rates

Fig. 8(a)-8(f) illustrate the BER versus data rate of 16-, 32- and 64-QAM OFDM downstream and upstream signals over the $10 \mathrm{~m} \mathrm{VLC}$ and $10 \mathrm{~km}$ fiber links. The dotted line at BER of $3.8 \times 10^{-3}$ presents the minimum BER value for the successful receipt of the signal under the forward error correction (FEC) of 7\% (Wei et al. 2017). From figures, it is observed that as the data rate increases, the BER of the downstream and upstream VLC signals increases for all three modulations. BER values increase due to the significant impacts of fiber nonlinearity at high data rate. Also, it can be observed that the VLC signal at $750 \mathrm{~nm}$ wavelength achieves superior performance over other wavelengths for all m-QAM modulations. This is because the VLC signal at $750 \mathrm{~nm}$ wavelength has high multipath fading resilience than other wavelengths. Further, it can be noticed that 16-QAM performs better than 32- and 64-QAM modulation formats. For the downstream and upstream 16QAM VLC signals, the minimum BER (at 7\% FEC limit) for better network performance is measured as $6.29 \times 10^{-4}$ and $6.32 \times 10^{-4}$ respectively at the transmission rate of $30 \mathrm{Gbps}$. Similarly, at the same acceptable limit, for 32-QAM modulation, the BER is measured as $1.62 \times 10^{-3}$ and $1.64 \times 10^{-3}$ at the information rate of $25 \mathrm{Gbps}$ whereas for 64-QAM modulation the BER is measured as $3.38 \times 10^{-3}$ and $3.68 \times 10^{-3}$ at 20 Gbps in the downstream and upstream directions respectively. The results observed demonstrate a reliable transmission of aggregate $120 \mathrm{Gbps}, 100 \mathrm{Gbps}$ and $80 \mathrm{Gbps}$ data over $10 \mathrm{~m}$ VLC and $10 \mathrm{~km}$ fiber links with acceptable BER $\left(3.8 \times 10^{-3}\right)$ for $16-$, 32- and 64-QAM modulations respectively in downstream and upstream directions. Moreover, Fig. 8 provides the constellation diagrams for 16-, 32- and 64-QAM modulation formats at 10 Gbps, 20 Gbps and $30 \mathrm{Gbps}$ data rates which illustrates that received signal performs better at a lower data rate.

Table 3 Comparative analysis of the proposed PON/VLC link over $10 \mathrm{~km}$ fiber distance with earlier works

\begin{tabular}{|c|c|c|c|c|c|}
\hline Reference & Technique used & $\begin{array}{c}\text { Modulation } \\
\text { format }\end{array}$ & $\begin{array}{c}\text { Maximum } \\
\text { wireless } \\
\text { range } \\
(\mathbf{m}) \text { @ BER } \\
\text { of 10-3 }\end{array}$ & $\begin{array}{c}\text { Maximum } \\
\text { SMF length } \\
(\mathbf{k m}) \text { @ BER } \\
\text { of 10 } \mathbf{1 0}^{-3}\end{array}$ & $\begin{array}{c}\text { Maximum } \\
\text { Data rate } \\
\text { (Gbps) } \\
\text { @ BER of } \\
\mathbf{1 0}^{-3}\end{array}$ \\
\hline $\begin{array}{c}\text { (Rahman et } \\
\text { al. 2020) }\end{array}$ & RoF-VLC & 3 & 10 & 2.5 \\
\hline $\begin{array}{c}\text { (Tang et al. } \\
\text { 2021) }\end{array}$ & $\begin{array}{c}\text { OFDM in hybrid fiber } \\
\text { - VLLC system }\end{array}$ & $\begin{array}{c}\text { 64- and } \\
\text { 128-QAM }\end{array}$ & 5 & 10 & 10 \\
\hline \multicolumn{2}{|c|}{ Proposed work } & $\begin{array}{c}\text { 16-, 32- and } \\
\text { 64-QAM }\end{array}$ & 10 & 10 & 10 \\
\hline
\end{tabular}


Table 3 illustrates that the previously demonstrated works in the literature deploy 16-, 64- and 128 QAM modulation formats capable of delivering maximum information only up to $10 \mathrm{~m} \mathrm{VLC}$ and $10 \mathrm{~km}$ fiber link reach at $10 \mathrm{Gbps}$ under the BER of $10^{-3}$. However, under the same BER, the higher information rate of $10 \mathrm{Gbps}$ over the wired-wireless links per channel (10 km wired and $10 \mathrm{~m}$ wireless) than previous work is obtained for the proposed TWDM PON system.

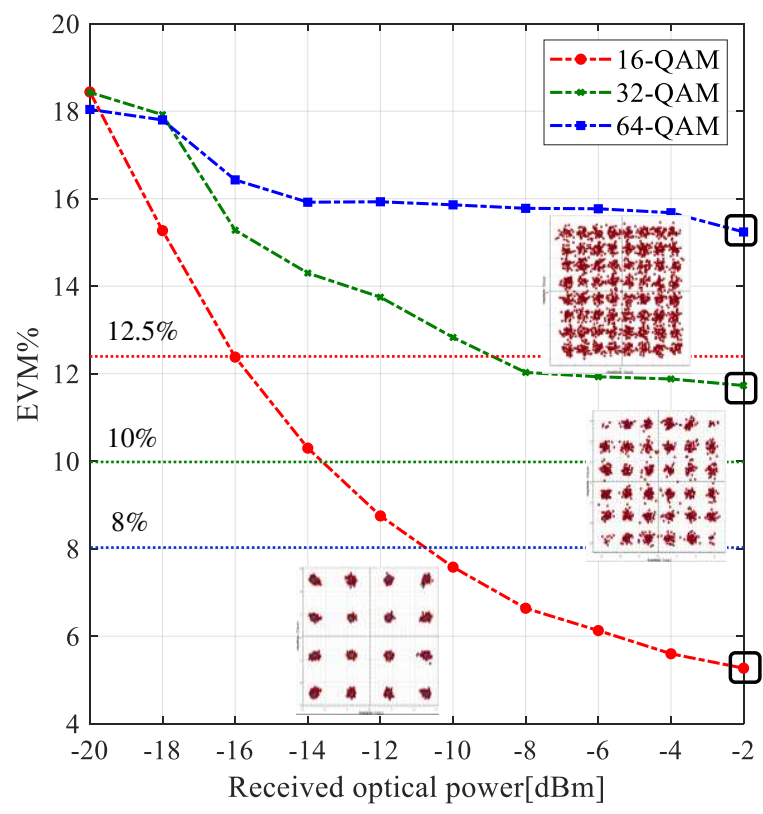

(a)

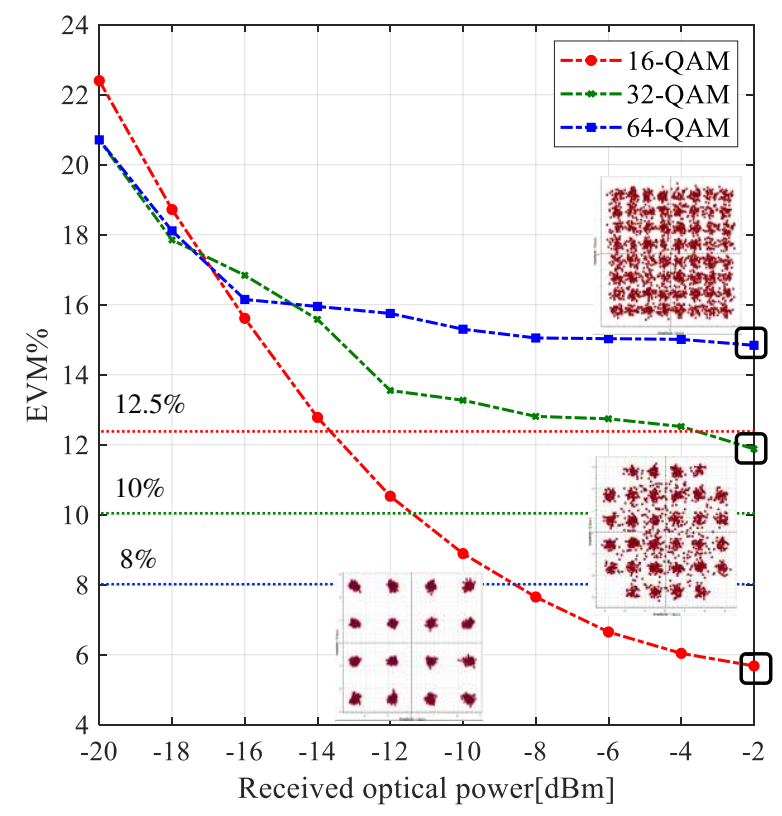

(b)

Fig. 9 EVM versus received optical power of 750nm wavelength VLC signal for (a) downstream 16-, 32- and 64-QAM and (b) upstream 16-, 32- and 64-QAM modulation formats. Insets show corresponding constellation diagram at $-2 \mathrm{dBm}$ received optical power

Fig. 9(a) and 9(b) present the EVM(\%) with respect to received optical power of 16-, 32- and 64-QAM OFDM downstream and upstream signals over the $10 \mathrm{~m} \mathrm{VLC} \mathrm{(at} 750 \mathrm{~nm}$ ) and $10 \mathrm{~km}$ fiber links at the information rate of $10 \mathrm{Gbps}$. EVM is utilized to measure the received signal quality under the impact of fiber distortion and is determined from its constellation diagram. EVM is generally illustrated in percentage (\%) and the EVM $(\%)$ of the received signals with the symbol sequence of $S$ and its decision of $[S\rfloor_{\mathrm{d}}$ is given as follows (Sheetal and Singh 2018):

$$
\operatorname{EVM}(\%)=\sqrt{\frac{\overline{\left|S-\lfloor S\rfloor_{d}^{2}\right|}}{\overline{\left|S_{d}\right|^{2}}}} \times 100 \%
$$

where $\overline{(\ldots)}$ indicates the mean value. From Fig. 9, it can be noticed that the $\operatorname{EVM}(\%)$ reduces with the increment in received optical power intorducing in the deflated error during information transmission. For the downstream direction, EVM(\%) for 16-QAM is measured as 18.44, 8.75 and 5.27 whereas for 32-QAM is measured as $18.43,13.75$ and 11.73 and for 64-QAM is measured as $18.04,15.93$ and 15.24 at the received optical power of $-20 \mathrm{dBm},-12 \mathrm{dBm}$ and $-2 \mathrm{dBm}$ respectively. Again, for upstream direction, EVM(\%) for 16-QAM is measured as 22.40, 10.53 and 5.68 whereas for 32-QAM is measured as 20.71, 13.55 and 11.88 and for 64-QAM is measured as 20.71, 15.75 and 14.84 at a received optical power of $-20 \mathrm{dBm},-12 \mathrm{dBm}$ and $-2 \mathrm{dBm}$ respectively. At $-20 \mathrm{dBm}$ received power, $\operatorname{EVM}(\%)$ values for both downstream and upstream signals are almost the same i.e. $18 \%$. After that, the EVMs between m-QAM signals are greatly separated EVM $(\%)$ in both transmission directions at $-2 \mathrm{dBm}$ received power and decreased by almost $12 \%$ for 16-QAM, 6\% for 32-QAM and $2 \%$ for 64-QAM according to the given $\operatorname{EVM}(\%)$ equation (20). Also, at the received optical power of $-2 \mathrm{dBm}$, the measured EVM for 16-QAM is below the required limit of $12.5 \%$ while for 32- and 64-QAM are above the required limits of $10 \%$ and $8 \%$ respectively with the reference of 3GPP specifications (Nguyen et al. 2019). Thus, it is observed that even at higher received power, high-order QAM modulation formats are more sensitive to noise. The minimal received optical powers required to fulfill the limits are $-16 \mathrm{dBm}$ and $-14 \mathrm{dBm}$ for 16 QAM VLC downstream and upstream signals respectively. However, for both 32- and 64-QAM VLC downstream and upstream signals, the required received power is more than $-2 \mathrm{dBm}$. Thus, for the proposed link, the 16-QAM modulation format shows better performance than other formats. Influenced by issues of inphase/quadrature-phase mismatches in the modulator/demodulator components due to gain, dc offset and phase 
differences, EVM shows rise in values in \%age and thus, degrade the system performance. Also, illustrated in insects are the constellation diagrams depicting the high-quality data transmission of 16-QAM followed by 32and 64-QAM modulation formats. Further, the calculated BER based on the EVMs for m-QAM are also evaluated as given in equation (21) (Nguyen et al. 2019):

$$
B E R_{m-Q A M}=\frac{2}{\log _{2}(m)}\left(1-\frac{1}{\sqrt{m}}\right) \cdot \operatorname{erfc}\left(\sqrt{\frac{3}{2(m-1)} \times \frac{1}{E V M^{2}}}\right)
$$

where $\operatorname{erfc}$ (.) means the complementary error function. Table 4 shows the calculated BER results for the proposed 16-, 32- and 64-QAM VLC downstream and upstream signals.

Table 4 Calculated BER results for the proposed work at a received power of $-2 \mathrm{dBm}$

\begin{tabular}{|c|c|c|c|c|}
\hline \multirow{2}{*}{ m-QAM } & \multicolumn{2}{|c|}{ Downstream } & \multicolumn{2}{c|}{ Upstream } \\
\cline { 2 - 5 } & EVM(\%) & BER & EVM(\%) & BER \\
\hline 16-QAM & 5.27 & $8.01 \times 10^{-18}$ & 5.68 & $1.29 \times 10^{-15}$ \\
\hline 32-QAM & 11.73 & $2.29 \times 10^{-3}$ & 11.88 & $2.90 \times 10^{-3}$ \\
\hline 64-QAM & 15.24 & $4.43 \times 10^{-2}$ & 14.84 & $4.12 \times 10^{-2}$ \\
\hline
\end{tabular}

The above-reported results show that the BER values are below the $7 \%$ FEC limit (at BER=3.08 $\times 10^{-3}$ ) for 16 QAM downstream and upstream signals. While for 32- and 64-QAM, the BER values are above the FEC limit in the bidirectional transmission. Hence, 16-QAM modulation is desirable for improving the performance of the proposed PON/VLC link.

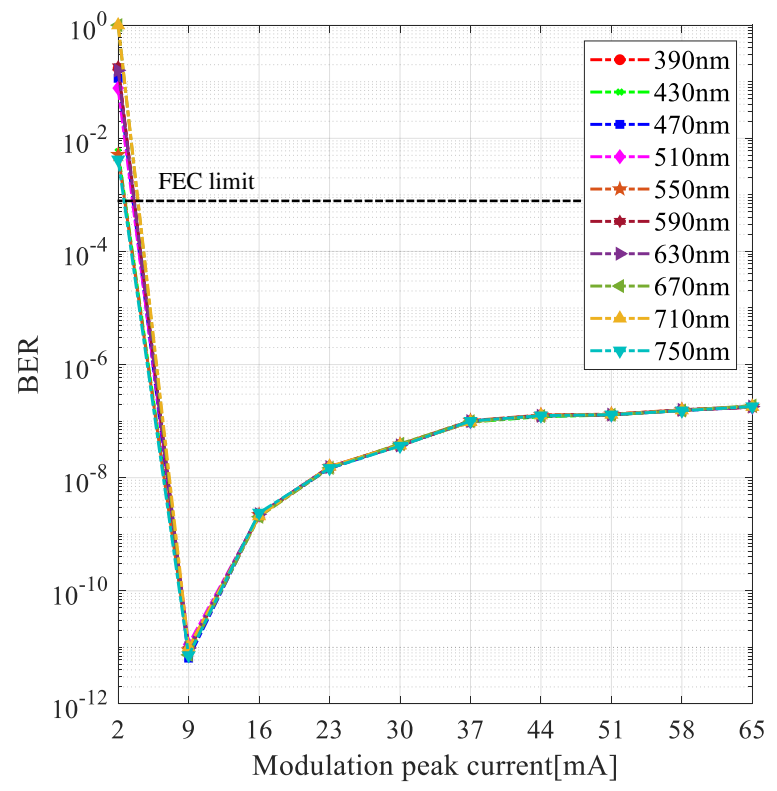

(a)

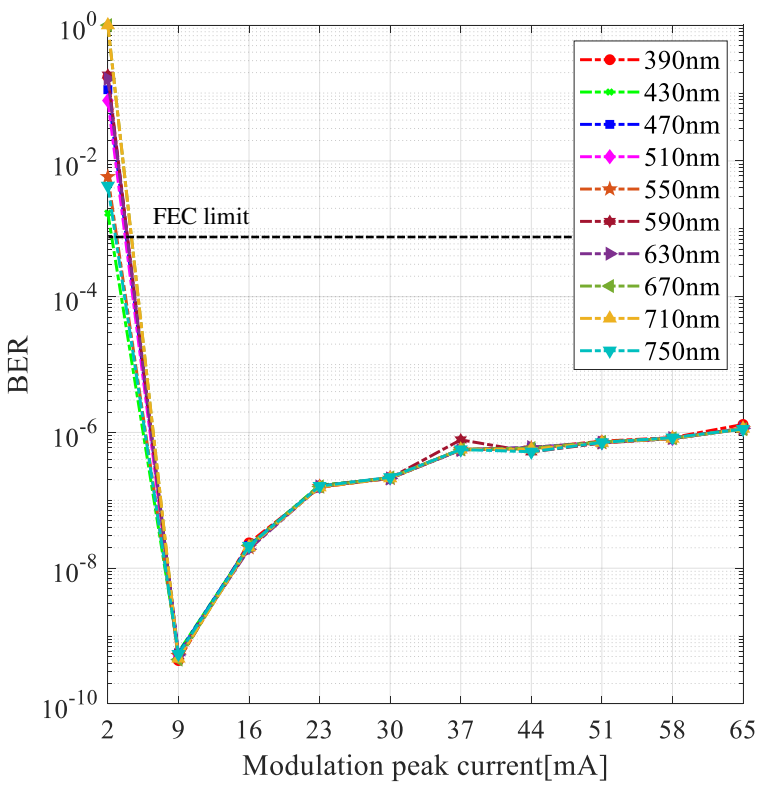

(b) 


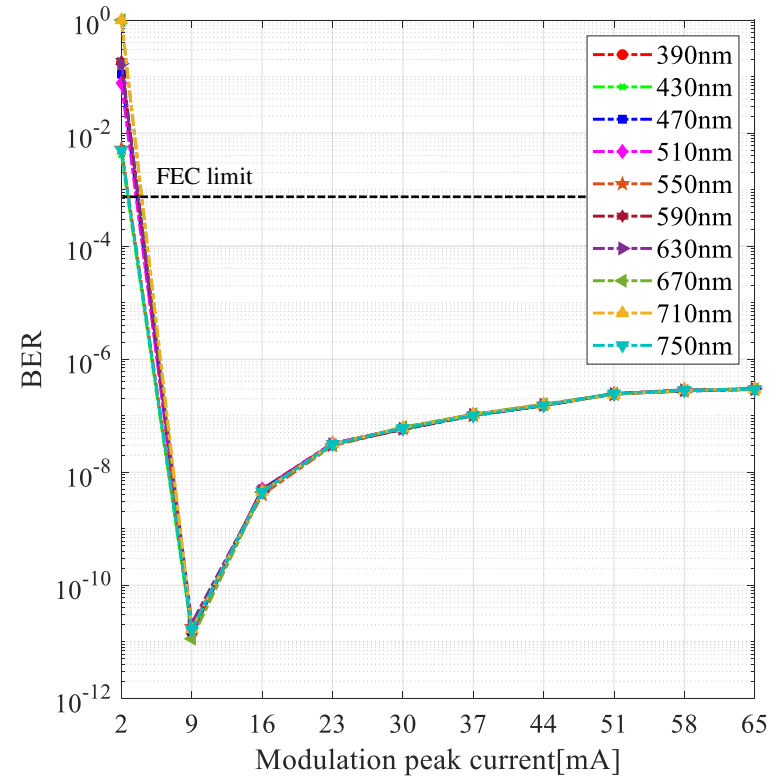

(c)

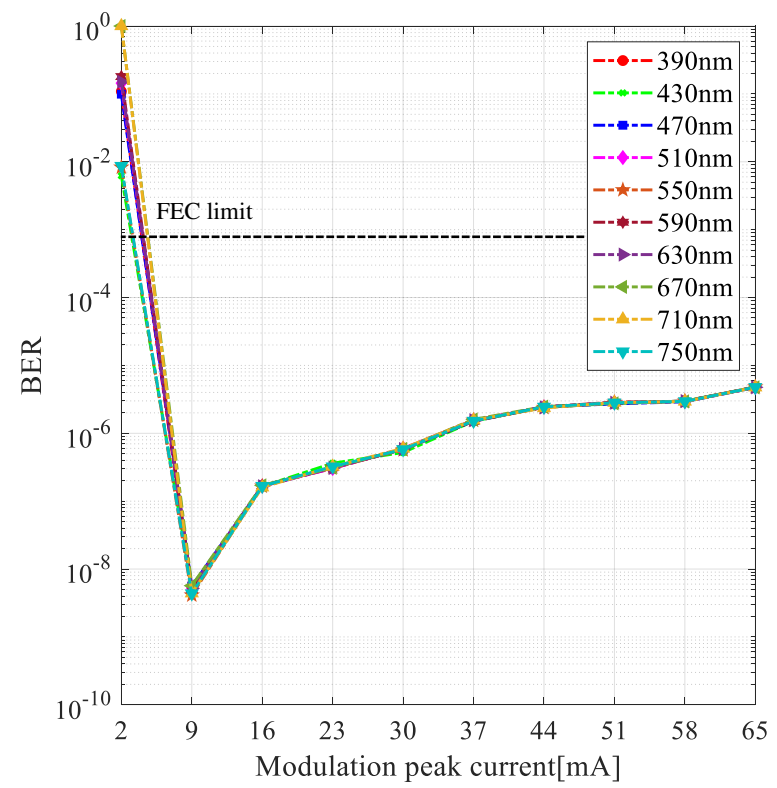

(e)

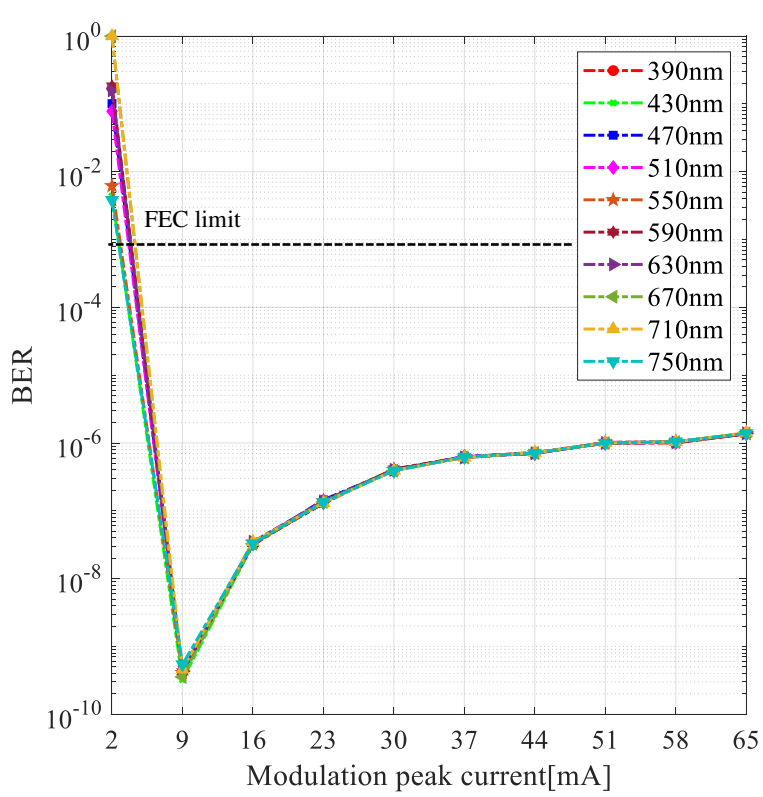

(d)

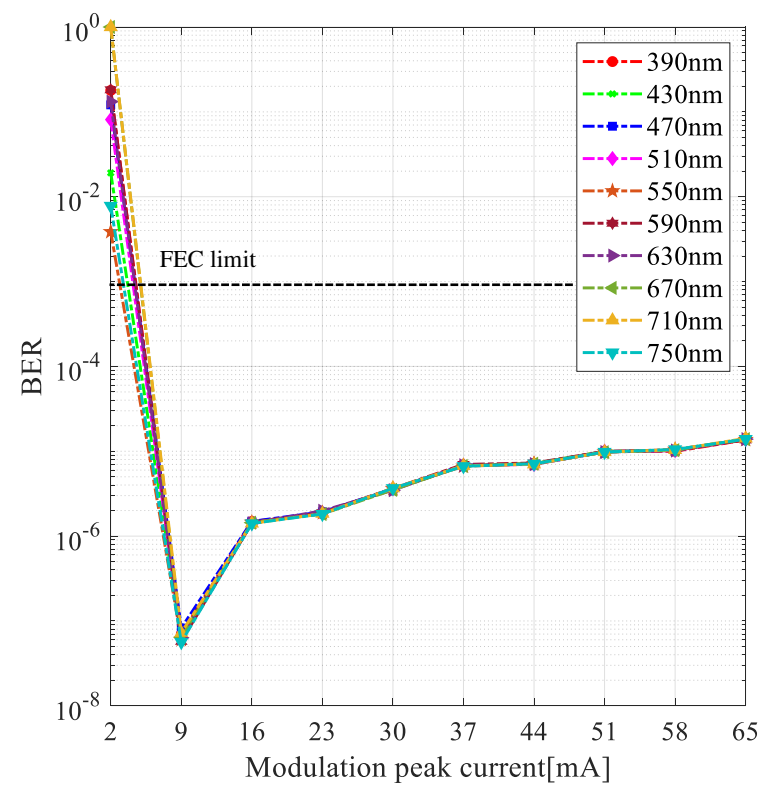

(f)

Fig. 10 Measured BER versus modulation peak current of different wavelengths VLC signals for (a) downstream 16-QAM, (b) upstream 16-QAM, (c) downstream 32-QAM, (d) upstream 32-QAM, (e) downstream 64-QAM and (f) upstream 64-QAM modulation formats

Fig. 10(a) to 10(f) present the BER versus modulation peak current of 16-, 32- and 64-QAM OFDM downstream and upstream signals over the fixed $8000 \mathrm{~m} \mathrm{VLC}$ and $50 \mathrm{~km}$ fiber links at the transmission rate of 10 Gbps. From Fig. 10 it is found that BER value decreases with the increase of input modulation peak current up to $9 \mathrm{~mA}$ for downstream/upstream signals beyond which it again increases. The BER value increases before $9 \mathrm{~mA}$, decrease at $9 \mathrm{~mA}$ and again increases after $9 \mathrm{~mA}$ because of the dominant under-modulation, peakmodulation and cross-modulation effects in the wireless links. Also, it can be observed that as the modulation type increases from 16- to 64-QAM, the BER of the downstream and upstream received signals for all wavelengths increases. In the downstream direction, the minimum obtained BER at a modulation current of $9 \mathrm{~mA}$ for 16-, 32- and 64-QAM modulation formats is measured as $6.46 \times 10^{-12}, 1.13 \times 10^{-11}$ and $4.12 \times 10^{-9}$ respectively. Also, in the upstream direction, the minimum obtained BER at a modulation peak current of $9 \mathrm{~mA}$ for all three modulation formats is measured as $4.36 \times 10^{-10}, 4.58 \times 10^{-10}$ and $5.63 \times 10^{-8}$ respectively. Thus, the obtained results demonstrate the best performance of the proposed TWDM-PON employing 16-QAM modulation format followed by 32- and 64-QAM modulation formats at 9mA modulation current set for individual wavelength LDs in the VLC system. 
Table 5 Comparative analysis of proposed PON/VLC link over $50 \mathrm{~km}$ fiber distance with previous works

\begin{tabular}{|c|c|c|c|c|c|}
\hline Reference & Technique used & $\begin{array}{c}\text { Modulation } \\
\text { format }\end{array}$ & $\begin{array}{c}\text { Maximum } \\
\text { wireless } \\
\text { range } \\
(\mathbf{k m}) \\
\text { @BER of } \\
\mathbf{1 0}^{-3} \\
\end{array}$ & $\begin{array}{c}\text { Maximum } \\
\text { SMF } \\
\text { length } \\
(\mathbf{k m}) \\
\text { @ BER of } \\
\mathbf{1 0}^{-3} \\
\end{array}$ & $\begin{array}{c}\text { Aggregate } \\
\text { Maximum } \\
\text { Data rate } \\
\text { (Gbps) } \\
\text { @BER of 10-3 }\end{array}$ \\
\hline $\begin{array}{l}\text { (Shi et al. } \\
\text { 2018) }\end{array}$ & $\begin{array}{l}\text { WDM fiber VLLC system based on } \\
\text { OFDM/OQAM }\end{array}$ & $\begin{array}{l}\text { 4- and 16- } \\
\text { QAM }\end{array}$ & 0.0045 & 50 & 3.2 \\
\hline $\begin{array}{c}\text { (Mallick et } \\
\text { al. 2019) }\end{array}$ & OFDM-FSO system & 16-QAM & 0.5 & 50 & 10 \\
\hline $\begin{array}{c}\text { (Singh and } \\
\text { Malhotra } \\
\text { 2019) }\end{array}$ & Hybrid MDM-OFDM-FSO syem & 4-QAM & 3.6 & 50 & 80 \\
\hline & Proposed work & $\begin{array}{c}\text { 16-, 32- and } \\
\text { 64-QAM }\end{array}$ & 8 & 50 & 120 \\
\hline
\end{tabular}

Table 5 illustrates that the previously work done in the literature deploy 4-, 16-, 32- and 64-QAM modulation formats capable of transferring data only up to highest of $3.6 \mathrm{~km}$ wireless and $50 \mathrm{~km}$ SMF range at the transmission rate of $80 \mathrm{Gbps}$. Whereas higher data rate of $120 \mathrm{Gbps}$ over long-reach wired-wireless links per channel of $8 \mathrm{~km}$ wireless and $50 \mathrm{~km}$ wired is obtained by using m-QAM OFDM modulation format and multicolor LDs based VLC system than previous work under BER of $10^{-3}$. Further, the power budget (PB) with receiver sensitivity at $10 \mathrm{dBm}$ input power of the proposed system for $8000 \mathrm{~m}$ VLC link using the following equation (22) is calculated in Table 6.

$$
P B=\text { Launch Power }-R S
$$

Table 6 Power Budget for the proposed system for $8000 \mathrm{~m}$ VLC link

\begin{tabular}{|c|c|c|}
\hline Channels & PB (dB) & RS (dBm) \\
\hline Downstream & 20 & -10 \\
\hline Upstream & 60 & -50 \\
\hline
\end{tabular}

Table 7 Comparative analysis of the proposed work with earlier recent works

\begin{tabular}{|c|c|c|c|c|c|c|}
\hline References & $\begin{array}{c}\text { Techniques } \\
\text { used }\end{array}$ & Modulation formats & $\begin{array}{l}\text { Maximum } \\
\text { wireless } \\
\text { range } \\
\text { (m) @ BER } \\
\text { of } 10^{-3}\end{array}$ & $\begin{array}{l}\text { Maximum } \\
\text { SMF length } \\
(\mathrm{km}) @ \text { BER } \\
\text { of } 10^{-3}\end{array}$ & $\begin{array}{c}\text { Maximum } \\
\text { Data rate } \\
\text { (Gbps) } \\
\text { @BER of } \\
\text { 10 }^{-3} \\
\end{array}$ & $\begin{array}{c}\text { Deployment } \\
\text { cost }\end{array}$ \\
\hline $\begin{array}{c}\text { (Shi et al. } \\
2018)\end{array}$ & $\begin{array}{c}\text { OFDM/OQA } \\
\text { M based } \\
\text { WDM fiber } \\
\text { VLLC system }\end{array}$ & 4- and 16-QAM & 4.5 & 50 & 3.2 & Medium \\
\hline $\begin{array}{c}\text { (Wei et al. } \\
\text { 2017) }\end{array}$ & $\begin{array}{l}\text { OFDM in } \\
\text { fiber-VLLC } \\
\text { system }\end{array}$ & 16-QAM & 8 & 20 & 2.5 & Medium \\
\hline $\begin{array}{c}\text { (He et al. } \\
2016 a)\end{array}$ & $\begin{array}{l}\text { DM-CAP- } \\
\text { PON } \\
\text { integration } \\
\text { with VLLC } \\
\text { system }\end{array}$ & 16-QAM & 8 & 30 & 16 & Medium \\
\hline $\begin{array}{c}\text { (Shaddad et } \\
\text { al. 2012) }\end{array}$ & $\begin{array}{c}\text { Wireless } \\
\text { MIMO } \\
\text { OFDM } \\
\text { signals over } \\
\text { WDM PON }\end{array}$ & 64-QAM & 100 & 20 & 7.68 & Medium \\
\hline (Mallick et & OFDM-FSO & 16-QAM & 500 & 50 & 10 & Low \\
\hline
\end{tabular}




\begin{tabular}{|c|c|c|c|c|c|c|}
\hline al. 2019) & system & & & & Low \\
\hline $\begin{array}{c}\text { (Singh and } \\
\text { Malhotra } \\
2019)\end{array}$ & $\begin{array}{c}\text { Hybrid } \\
\text { MDM- } \\
\text { OFDM-FSO } \\
\text { syem }\end{array}$ & 4-QAM & 3600 & 50 & 80 & \\
\hline \multicolumn{2}{|c|}{ Proposed work } & 16-, 32- and 64-QAM & 8000 & 50 & 120 & Medium \\
\hline
\end{tabular}

In Table 7 the proposed system shows that the previously reported works deploy 4-, 16- and 64-QAM modulation formats are capable of sending information only up to $3600 \mathrm{~m}$ wireless and 50km fiber link range at the transmission rate of $80 \mathrm{Gbps}$ with low/medium deployment cost. While the high information rate over fiber/VLC links per channel than previous work is received by using 16-, 32- and 64-QAM OFDM modulation format and multi-color LDs based VLC system in the proposed PON/VLC link at medium deployment cost. It shows that the proposed TWDM PON system offers high data rate, efficient bandwidth utilization by supporting the large number of customers with cost-effectiveness, reliable, scalable, flexible and easy upgradeable features.

\section{Conclusion}

In this paper, a full-duplex $4 \times 10$ Gbps TWDM-PON utilizing 16-, 32- and 64-QAM OFDM modulation formats with ten different colors LDs enabled VLC system has been demonstrated. From results, it is concluded that the faithful wireless range in PON/VLC link varies from $10 \mathrm{~m}$ to $8000 \mathrm{~m}$ at a fixed fiber length of $50 \mathrm{~km}$ and $9 \mathrm{~mA}$ LD input modulation peak current with minimum acceptable BER of $3.8 \times 10^{-3}$. Also, the highest achieved transmission rates for the designed system employing m-QAM modulation with $10 \mathrm{~m}$ free space and $10 \mathrm{~km}$ fiber link range are $120 \mathrm{Gbps}$ for 16-QAM, $100 \mathrm{Gbps}$ for 32-QAM and $80 \mathrm{Gbps}$ for 64-QAM in both downstream and upstream directions. Moreover, it is investigated that for 16-QAM modulation, the recovered downstream and upstream signals show measured EVM and calculated BER values are lower than the FEC threshold as defined by 3GPP specifications. Again, the correlated performance of the proposed system performance with the recent previous literature indicates the significant superiority for the maximum transmission length and data rate. Hence, the system can be utilized to offer economic and high-speed fiber/wireless link based applications for the $5 \mathrm{G}$ based applications.

\section{References}

Alqahtani, A.H., Humadi, K., Sulyman, A.I., Alsanie, A.: Experimental Evaluation of MIMO-OFDM System with Rateless Space-Time Block Code. Int. J. Antennas Propag. 2019, 1-8 (2019)

Anis, M.I., Qureshi, M.S., Zafar, S.: Demonstration of TWDM-PON Backward Compatibility with Conventional GPON. Wirel. Pers. Commun. 95, 581-592 (2017)

Bai, W., Zou, X., Li, P., Pan, W., Yan, L., Luo, B., Lu, X.: A WDM-PON compatible wavelength-reused bidirectional in-band full-duplex radio-over-fiber system. Opt. Commun. 463, 125408 (2020). https://doi.org/10.1016/j.optcom.2020.125408

Bi, M., Zhuo, X., Yang, G., Hu, M., Fan, B., Yang, X., Hu, W.: Chaotic Arnold transform and chirp matrix encryption scheme for enhancing the performance and security of OFDM-PON. Opt. Fiber Technol. 51, 64-70 (2019). https://doi.org/10.1016/j.yofte.2019.05.005

Chen, C., Zhong, W.-D., Wu, D.: Integration of variable-rate OWC with OFDM-PON for hybrid optical access based on adaptive envelope modulation. Opt. Commun. 381, 10-17 (2016)

Chen, M., Lu, H., Chen, D., Jin, J., Wang, J.: An efficient MIMO-OFDM VLC system of combining space time block coding with orthogonal circulant matrix transform precoding. Opt. Commun. 473, 125993 (2020). https://doi.org/10.1016/j.optcom.2020.125993

Chen, Q., Bi, M., Fu, X., Lu, Y., Zeng, R., Yang, G., Yang, X., Xiao, S.: Security scheme in IMDD-OFDMPON system with the chaotic pilot interval and scrambling. Opt. Commun. 207, 285-289 (2018)

Chi, N., Shi, J.: Investigation on overlapping interference on VLC networks consisting of multiple LEDs. ICT Express. 1, 63-66 (2015). https://doi.org/10.1016/j.icte.2015.09.004

Choudhury, P.K.: Enhanced noise tolerance for $10 \mathrm{~Gb} / \mathrm{s}$ Bi-directional cross-wavelength reuse colorless WDMPON by using spectrally shaped OFDM signals. Opt. Fiber Technol. 42, 6-10 (2018)

Chow, C.W., Yeh, C.H., Liu, Y.F., Huang, P.Y., Liu, Y.: Adaptive scheme for maintaining the performance of the in-home white-LED visible light wireless communications using OFDM. Opt. Commun. 292, 49-52 (2013). https://doi.org/10.1016/j.optcom.2012.11.081

Deng, L., Pang, X., Zhao, Y., Othman, M.B., Jensen, J.B., Zibar, D., Yu, X., Liu, D., Monroy, I.T.: 2x2 MIMO- 
OFDM Gigabit fiber-wireless access system based on polarization division multiplexed WDM-PON. Opt. Express. 20, 4369-4375 (2012)

Deng, R., He, J., Chen, M., Zhou, Y.: Experimental Demonstration of a Real-Time gigabit OFDM-VLC system with a cost-efficient precoding scheme. Opt. Commun. 423, 69-73 (2018). https://doi.org/10.1016/j.optcom.2018.04.009

Fusheng, L., Ruisheng, L., Fengquan, Z.: Communication of the microgrid. In: Microgrid Technology and Engineering Application. pp. 115-124 (2016)

Gill, H.K., Walia, G.K., Grewal, N.S.: Performance analysis of mode division multiplexing IS-OWC system using Manchester, DPSK and DQPSK modulation techniques. Optik (Stuttg). 177, 93-101 (2019). https://doi.org/10.1016/j.ijleo.2018.09.032

Grover, A., Sheetal, A.: A cost-effective high-capacity OFDM based RoFSO transmission link incorporating hybrid SS-WDM-MDM of Hermite Gaussian modes. Optoelectron. Adv. Mater. - RAPID Commun. 14, 136145 (2020)

Grover, A., Sheetal, A., Dhasarathan, V.: Performance analysis of mode division multiplexing based free space optics system incorporating on-off keying and polarization shift keying under dynamic environmental conditions. Wirel. Networks. 26, 3439-3449 (2020). https://doi.org/10.1007/s11276-020-02275-6

Guerreiro, J., Dinis, R., Campos, L.: On the Achievable Capacity of MIMO-OFDM Systems in the CathLab Environment. Sensors. 20, 938 (2020). https://doi.org/10.3390/s20030938

He, J., Dong, H., Deng, R., Chen, L.: Low-cost bidirectional hybrid fiber-visible laser light communication system based on carrier-less amplitude phase modulation. Opt. Eng. 55, 086109 (2016)(a). https://doi.org/10.1117/1.oe.55.8.086109

He, J., Dong, H., Deng, R., Shi, J., Chen, L.: WDM-CAP-PON integration with VLLC system based on optical frequency comb. Opt. Commun. 374, 127-132 (2016)(b). https://doi.org/10.1016/j.optcom.2016.04.059

$\mathrm{Hu}, \mathrm{Z}$., Qiu, Y., Li, W., Chan, C.-K.: Experimental demonstration of direct re-modulation for an IM/DD OFDM-WDM-PON with symmetrical bi-directional transmission. Opt. Commun. 460, 125123 (2020)

Huang, Y., Chen, Y., Li, K., Han, Y., Fu, J., Li, Y., Ma, J., Yu, J., Li, X.: Multi scrolls chaotic encryption for physical layer security in OFDM-PON. Opt. Commun. 471, 126009 (2020). https://doi.org/10.1016/j.optcom.2020.126009

Jani, M., Garg, P., Gupta, A.: Performance Analysis of a Co-Operative PLC/VLC System with Multiple Access Points for Indoor Broadcasting. AEU - Int. J. Electron. Commun. 103, 64-73 (2019). https://doi.org/10.1016/j.aeue.2019.02.012

Ju, C., Liu, N., Chen, X.: Iteration SSII cancellation in DD-OFDM PON upstream scheme. Opt. Commun. 364, 9-12 (2016). https://doi.org/10.1016/j.optcom.2015.11.014

Kachhatiya, V., Prince, S.: Four-fold increase in users of time-wavelength division multiplexing (TWDM) passive optical network (PON) by delayed optical amplitude modulation (AM) upstream. Opt. Fiber Technol. 32, 71-81 (2016)

Kachhatiya, V., Prince, S.: Downstream performance analysis and optimization of the next generation passive optical network stage 2 (NG-PON2). Opt. Laser Technol. 104, 90-102 (2018). https://doi.org/10.1016/j.optlastec.2018.02.007

Kartiwa, I., Jung, S.-M., Hong, M.-K., Han, S.-K.: 20Gb/s WDM-OFDM-PON over 20-km single fiber uplink transmission using optical millimeter-wave signal seeding with rate adaptive bit-power loading. Opt. Fiber Technol. 19, 231-235 (2013)

Kaur, A., Kaur, B., Singh, K.: Design and performance analysis of bidirectional TWDM-PON employing QAM-OFDM for downstream and re-modulation for upstream. Optik (Stuttg). 134, 287-294 (2017)(a). https://doi.org/10.1016/j.ijleo.2017.01.009

Kaur, A., Sheetal, A., Miglani, R.: Impact of optical modulation formats on 10 G/2.5 G asymmetric XG-PON system. Optik (Stuttg). 149, 351-358 (2017)(b). https://doi.org/10.1016/j.ijleo.2017.09.063

Kaur, A., Singh, M.L., Sheetal, A.: Simulative analysis of co-existing 2.5 G/10 G asymmetric XG-PON system using RZ and NRZ data formats. Optik (Stuttg). 125, 3637-3640 (2014). https://doi.org/10.1016/j.ijleo.2014.01.074

Kumari, M., Sharma, R., Sheetal, A.: Comparative Analysis of High Speed 20/20 Gbps OTDM-PON, WDMPON and TWDM-PON for Long-Reach NG-PON2. J. Opt. Commun. 0, 1-14 (2019). https://doi.org/10.1515/joc-2019-0005

Kumari, M., Sharma, R., Sheetal, A.: Performance analysis of high speed backward compatible TWDM-PON with hybrid WDM-OCDMA PON using different OCDMA codes. Opt. Quantum Electron. 52, (2020)

Lee, S., Jeon, Y., Lim, T., Lee, K., Park, J.: A wireless access network based on WDM-PON for HMIPv6 mobility support. Wirel. Networks. 16, 1707-1722 (2010). https://doi.org/10.1007/s11276-009-0223-9

Li, H., Zhang, Y., Chen, X., Wu, C., Guo, J., Gao, Z., Pei, W., Chen, H.: 682 Mbit/s phosphorescent white LED visible light communications utilizing analog equalized 16QAM-OFDM modulation without blue filter. Opt. Commun. 354, 107-111 (2015). https://doi.org/10.1016/j.optcom.2015.05.033 
Lin, B., Li, Y., Zhang, S., Tang, X.: Asymmetrical TWDM-PON with 4×25-Gb/s downstream DSB OFDM and $4 \times 10-\mathrm{Gb} / \mathrm{s}$ upstream OOK modulations. Opt. Fiber Technol. 26, 206-210 (2015). https://doi.org/10.1016/j.yofte.2015.09.007

Lu, L., Li, G.Y., Swindlehurst, A.L., Ashikhmin, A., Zhang, R.: An Overview of Massive MIMO : Bene fi ts and Challenges. IEEE J. Sel. Top. Signal Process. 8, 742-758 (2014)

Lu, M., Xiao, S., Zhang, L., Zheng, L., Fang, J., Huang, T., Hu, W.: Real-time VLC system integrated with positioning beacon transmission based on 2ASK-CE-OFDM coding. Opt. Commun. 452, 252-257 (2019). https://doi.org/10.1016/j.optcom.2019.07.066

Lyu, W.C., Wang, A., Xie, D., Zhu, L., Guan, X., Wang, J., Xu, J.: Self-homodyne optical OFDM for broadband WDM-PONs with crosstalk-free remodulation and enhanced tolerance to Rayleigh noise. Opt. Commun. 414, 77-82 (2018). https://doi.org/10.1016/j.optcom.2018.01.003

Ma, J., He, J., Chen, Q., Shi, J., Zhou, Z., Cheng, Y., Xiao, Y.: A MB-CAZAC precoding combined with 128/64/32/16-QAM modulation for OFDM-VLC system. Opt. Commun. 424, 154-158 (2018). https://doi.org/10.1016/j.optcom.2018.04.047

Mallick, K., Mandal, P., Mandal, G.C., Mukherjee, R., Das, B., Patra, A.S.: Hybrid MMW-over fiber/OFDMFSO transmission system based on doublet lens scheme and POLMUX technique. Opt. Fiber Technol. 52, 101942 (2019). https://doi.org/10.1016/j.yofte.2019.101942

Mallick, K., Mandal, P., Mukherjee, R., Mandal, G.C., Das, B., Patra, A.S.: Generation of 40 GHz/80 GHz OFDM based MMW source and the OFDM-FSO transport system based on special fine tracking technology. Opt. Fiber Technol. 54, 102130 (2020). https://doi.org/10.1016/j.yofte.2019.102130

Mallick, K., Mukherjee, R., Das, B., Chandra, G., Sekhar, A.: Bidirectional hybrid OFDM based Wireless-overfiber transport system using reflective semiconductor amplifier and polarization multiplexing technique. AEU Int. J. Electron. Commun. 96, 260-266 (2018)

Mandal, G.C., Mukherjee, R., Das, B., Patra, A.S.: A full-duplex WDM hybrid fiber-wired/fiber-wireless/fiberVLC/fiber-IVLC transmission system based on a self-injection locked quantum dash laser and a RSOA. Opt. Commun. 427, 202-208 (2018)

Mandal, G.C., Patra, A.S.: High capacity hybrid WDM/TDM-PON employing fiber-to-the-home for triple-play services with 128 ONUs. J. Opt. 46, 347-351 (2017). https://doi.org/10.14569/IJACSA.2011.020818

Mandal, P., Mallick, K., Dutta, B., Kuiri, B., Santra, S., Patra, A.S.: Mitigation of Rayleigh backscattering in RoF WDM PON employing self coherent detection and bi directional cross wavelength technique. Opt. Quantum Electron. 53, (2021)

Mohd Nor, N.A., Ghassemlooy, Z., Zvanovec, S., Khalighi, M.A., Bhatnagar, M.R., Bohata, J., Komanec, M.: Experimental analysis of a triple-hop relay-assisted FSO system with turbulence. Opt. Switch. Netw. 33, 194198 (2019). https://doi.org/10.1016/j.osn.2017.11.002

Mukherjee, R., Mallick, K., Mandal, P., Dutta, B., Kuiri, B., Patra, A.S.: Bidirectional hybrid OFDM based free-space/wireless-over-fiber transport system. Opt. Quantum Electron. 52, (2020)

Nguyen, D.-N., Bohata, J., Spacil, J., Dousek, D., Komanec, M., Zvanovec, S., Ghassemlooy, Z., Ortega, B.: M-QAM transmission over hybrid microwave photonic links at the K-band. Opt. Express. 27, 33745 (2019). https://doi.org/10.1364/oe.27.033745

Omella, M., Polo, V., Lazaro, J., Schrenk, B., Prat, J.: 10 Gb/s RSOA transmission by direct duobinary modulation. In: European Conference on Optical Communication, ECOC. pp. 123-124 (2008)

Patel, D., Dalal, U.D.: A novel wavelength reused bidirectional RoF-WDM-PON architecture to mitigate reflection and Rayleigh backscattered noise in multi-Gb/s m-QAM OFDM SSB upstream and downstream transmission over a single fiber. Opt. Commun. 390, 26-35 (2017)

Prabu, K., Charanya, S., Jain, M., Guha, D.: BER analysis of SS-WDM based FSO system for Vellore weather conditions. Opt. Commun. 403, 73-80 (2017). https://doi.org/10.1016/j.optcom.2017.07.012

Rahman, M.T., Parthiban, R., Bakaul, M.: Integration and Evaluation of Hybrid RoF-VLC Network. In: 2020 IEEE 8th International Conference on Photonics (ICP). pp. 84-85 (2020)

Renaudier, J., Bertran-pardo, O., Charlet, G., Salsi, M., Mardoyan, H., Tran, P., Bigo, S.: 8 Tb / s Long Haul Transmission Over Low Dispersion Fibers Using $100 \mathrm{~Gb} / \mathrm{s}$ PDM-QPSK Channels Paired With Coherent Detection. Bell Labs Tech. J. 14, 27-45 (2010). https://doi.org/10.1002/bltj

Saraereh, O.A., Khan, I., Alsafasfeh, Q., Alemaishat, S., Kim, S.: Low-Complexity Channel Estimation in 5G Massive MIMO-OFDM Systems. Symmetry (Basel). 11, 713 (2019). https://doi.org/10.3390/sym11050713

Savojbolaghchi, H., Sadough, S.M.S., Dabiri, M.T., Ansari, I.S.: Generalized channel estimation and data detection for MIMO multiplexing FSO parallel channels over limited space. Opt. Commun. 452, 158-168 (2019). https://doi.org/10.1016/j.optcom.2019.07.017

Selvendran, S., Sivanantha Raja, A., Esakki Muthu, K., Lakshmi, A.: Certain Investigation on Visible Light Communication with OFDM Modulated White LED Using Optisystem Simulation. Wirel. Pers. Commun. 109, 1377-1394 (2019). https://doi.org/10.1007/s11277-019-06617-2

Shaddad, R.Q., Mohammad, A.B., Al-Hetar, A.M.: Spectral efficient hybrid wireless optical broadband access 
network (WOBAN) based on transmission of wireless MIMO OFDM signals over WDM PON. Opt. Commun. 285, 4059-4067 (2012). https://doi.org/10.1016/j.optcom.2012.06.002

Shao, Y., Chen, F., Wang, A., Luo, Y., Chen, L.: Application research on 4-pulsed amplitude modulation in 10 $\mathrm{Gb} / \mathrm{s}$ passive optical access systems. Optik (Stuttg). 146, $63-68 \quad$ (2017). https://doi.org/10.1016/j.ijleo.2017.08.072

Sheetal, A., Singh, H.: $5 \times 10$ Gbps WDM-CAP-PON based on frequency comb using OFDM with blue LD. Opt. Quantum Electron. 50, 1-14 (2018)

Shi, J., He, J., Zhang, R., Deng, R., Xiao, Y.: OFDM/OQAM based WDM fiber VLLC system employing improved channel estimation method. Opt. Commun. 427, 578-583 (2018). https://doi.org/10.1016/j.optcom.2018.07.034

Singh, M., Malhotra, J.: Long-Reach High-Capacity Hybrid MDM-OFDM-FSO Transmission Link Under the Effect of Atmospheric Turbulence. Wirel. Pers. Commun. 107, 1549-1571 (2019). https://doi.org/10.1007/s11277-019-06345-7

Tang, Q., Li, K., Liu, X., Kong, L.: Performance Analysis of Spectral Efficiently Adaptive Modulation DFTSpread Polar Coordinate-Based OFDM in Hybrid Fiber-Visibl .... In: 2020 IEEE 20th International Conference on Communication Technology (ICCT) (2021)

Wei, Y., He, J., Deng, R., Shi, J., Chen, S., Chen, L.: An approach enabling adaptive FEC for OFDM in fiberVLLC system. Opt. Commun. 405, 329-333 (2017). https://doi.org/10.1016/j.optcom.2017.08.041

Wu, T.C., Chi, Y.C., Wang, H.Y., Tsai, C.T., Huang, Y.F., Lin, G.R.: Tricolor R/G/B laser diode based eyesafe White lighting communication beyond 8 Gbit/s. Sci. Rep. 7, 1-10 (2017). https://doi.org/10.1038/s41598017-00052-8

Xiao, Y., Wang, Z., Cao, J., Long, C., Chen, Y., Deng, R., Shi, J., Liu, Y., He, J.: Two-level encryption for physical-layer security in OFDM-PON based on multi-scrolls system. Opt. Commun. 440, 126-131 (2019). https://doi.org/10.1016/j.optcom.2019.02.033

Xiao, Y., Yu, J.: A novel WDM-ROF-PON architecture based on 16QAM-OFDM modulation for bidirectional accessnetworks. Opt. Commun. 295, 99-103 (2013)

$\mathrm{Xu}$, T.: Digital Signal Processing for Optical Communications and Networks. In: Optical Fiber and Wireless Communications (2017)

Yang, H., Li, J., Lin, B., Wan, Y., Guo, Y., Zhu, L., Li, L., He, Y., Chen, Z.: DSP-based evolution from conventional TDM-PON to TDM-OFDM-PON. J. Light. Technol. 31, 3035-3041 (2013). https://doi.org/10.1109/jlt.2013.2271909

Yeh, C.H., Chow, C.W.: Utilization of four WDM channels with signal remodulation of OFDM-QAM for 10Gb/s uplink passive optical networks. Opt. Commun. 282, 3701-3705 (2009)

Yeh, C.H., Chow, C.W., Chen, H.Y., Liu, Y.L.: 115 Gbit/s downstream and 10 Gbit/s upstream TWDM-PON together with $11.25 \mathrm{Gbit} / \mathrm{s}$ wireless signal utilizing OFDM-QAM modulation. Opt. Fiber Technol. 20, 84-89 (2014). https://doi.org/10.1016/j.yofte.2013.12.004

Yeh, C.H., Chow, C.W., Gu, C.S., Guo, B.S., Cheng, Y.J., Chen, J.H.: Performance analysis of free space optical communication traffic integrated with passive optical network. Electron. Lett. 54, 1228-1229 (2018). https://doi.org/10.1049/el.2018.5559

Zhang, J., Hong, X., Liu, J., Guo, C.: Experimental demonstration of an OFDM based visible light communication system using inter-block precoding and superimposed pilots. Opt. Commun. 412, 219-225 (2018). https://doi.org/10.1016/j.optcom.2017.12.020

Zhang, W.F., Xin, X.J., Zhang, Q., Zhang, Z.X., Nai, W., Shi, Y.: Centralized light-wave WDM-PON employing DQPSK downstream and OOK remodulated upstream signals. J. China Univ. Posts Telecommun. 17, 125-128 (2010). https://doi.org/10.1016/S1005-8885(09)60498-2

Zhuo, X., Bi, M., Hu, Z., Li, H., Wang, X., Yang, X.: Secure scheme for OFDM-PON system using TR based on modified Henon chaos. Opt. Commun. 462, 125304 (2020) 